\title{
AN ANALYTIC PROOF OF RIEMANN-ROCH- HIRZEBRUCH THEOREM FOR KAEHLER MANIFOLDS
}

\author{
V. K. PATODI
}

\section{Introduction}

Let $X$ be a compact complex manifold of (complex) dimension $n$, and $\xi$ a holomorphic vector bundle over $X$. We shall denote by $\Omega(\xi)$ the sheaf of germs of holomorphic sections of $\xi$, and by $H^{i}(X, \Omega(\xi))$ the $i$-th cohomology group of the space $X$ with coefficients in the sheaf $\Omega(\xi)$. Then $H^{i}(X, \Omega(\xi))$ are finite dimensional vector spaces over the field $C$ of complex numbers, and $H^{i}(X, \Omega(\xi))=0$ for $i>n$. Let $\operatorname{dim} H^{i}(X, \Omega(\xi))$ denote the dimension of the vector space $H^{i}(X, \Omega(\xi))$, and $\chi(X, \Omega(\xi))$ be the Euler-Poincare characteristic defined by the formula

$$
\chi(X, \Omega(\xi))=\sum_{i=0}^{n}(-1)^{i} \operatorname{dim} H^{i}(X, \Omega(\xi)) .
$$

Let $\mathscr{T}(X)$ be the Todd class of the complex tangent boundle $T(X)$ of $X$, and $\operatorname{ch}(\xi)$ the Chern character of the holomorphic vector bundle $\xi$. Then the Riemann-Roch-Hirzebruch theorem can be stated as follows.

Theorem 1.1. The Euler-Poincaré characieristic $\chi(X, \Omega(\xi))$ can be expressed in terms of ch $(\xi)$ and $\mathscr{T}(X)$ :

$$
\chi(X, \Omega(\xi))=[\operatorname{ch}(\xi) \mathscr{T}(X)]_{2 n}[X] .
$$

Formula (1.1) can be interpreted as follows: $\operatorname{ch}(\xi)$ and $\mathscr{T}(X)$ are elements of $H^{*}(X, Z) \otimes \mathbf{Q}$. If the multiplication is considered as the cup product, then ch $(\xi) \mathscr{T}(X)$ defines an element of $H^{*}(X, Z) \otimes \mathbf{Q}$, and hence its $2 n$-th component defines an element of $H^{2 n}(X, Z) \otimes \mathbf{Q}$. The value of this element on the $2 n$-dimensional cycle of $X$ determined by the natural orientation is equal to $\chi(X, \Omega(\xi))$.

In this paper we shall give an analytic proof of this theorem under the assumption that $X$ is a Kaehler manifold. We start with the following observations. Let $\eta$ denote the complex vector bundle $\wedge\left(T^{*}(X)\right) \otimes C, T^{*}(X)$ being the cotangent bundle of $X$. Then $\eta$ has a canonical direct sum decomposition

Communicated by B. Kostant, May 5, 1970. 


$$
\eta=\bigoplus_{p, q} \eta^{p, q}
$$

$\eta^{p, q}$ being the sub-bundle of differential forms of type $(p, q)$ with values in the vector bundle $\xi$.

Let $\zeta^{q}=\xi \otimes \eta^{0, q}$ and $\zeta=\bigoplus_{q} \zeta^{q}$. Then there is a canonical operator $d_{\bar{z}}$ (exterior differentiation with respect to $\bar{z}$ ) from $C^{\infty}\left(\zeta^{q}\right) \rightarrow C^{\infty}\left(\zeta^{q+1}\right), 0 \leq q \leq n$. The following theorem of Dolbeault is the complex analogue of de Rham's theorem.

Theorem 1.2. Consider the complex

$$
0 \longrightarrow C^{\infty}\left(\zeta^{0}\right) \stackrel{d_{\bar{z}}}{\longrightarrow} C^{\infty}\left(\zeta^{1}\right) \stackrel{d_{\bar{z}}}{\longrightarrow} \cdots \stackrel{d_{\bar{z}}}{\longrightarrow} C^{\infty}\left(\zeta^{n}\right) \longrightarrow 0,
$$

and let $Z_{p}=\operatorname{kernel}\left(d_{\bar{z}}: C^{\infty}\left(\zeta^{q}\right) \rightarrow C^{\infty}\left(\zeta^{q+1}\right)\right)$ and $B_{q}=$ image $\left(d_{\bar{z}}: C^{\infty}\left(\zeta^{q-1}\right) \rightarrow\right.$ $\left.C^{\infty}(\zeta)\right), 0 \leq q \leq n$. Then the cohomology groups $Z_{q} / B_{q}$ are canonically isomorphic to the sheaf theoretic cohomology groups $H^{q}(X, \Omega(\xi))$.

We introduce hermitian metrices in the bundles $\xi$ and $T(X)$. Then there are canonical hermitian metrices in the bundles $\zeta^{q}, 0 \leq q \leq n$. Let $d_{\bar{z}}^{*}: C^{\infty}\left(\zeta^{q+1}\right)$ $\rightarrow C^{\infty}\left(\zeta^{q}\right)$ be the adjoint of the differential operator $d_{\bar{z}}: C^{\infty}\left(\zeta^{q}\right) \rightarrow C^{\infty}\left(\zeta^{q+1}\right)$ with respect to the hermitian metrices in the bundles $\zeta^{q}, \zeta^{q+1}$, and let $\zeta^{e}=\underset{q}{\oplus} \zeta^{2 q}$ and $\zeta^{0}=\bigoplus_{q} \zeta^{2 q+1}$. Then the operator $d_{\bar{z}}+d^{*}{ }_{\bar{z}}$ maps $C^{\infty}\left(\zeta^{e}\right)$ into $C^{\infty}\left(\zeta^{0}\right)$ and is easily seen to be an elliptic operator. The following proposition is an immediate consequence of Theorem 1.2 and the complex analogue of the Hodge decomposition theorem.

Proposition 1.3. The analytic index of the operator

$$
d_{\bar{z}}+d_{\bar{z}}^{*}: C^{\infty}\left(\zeta^{e}\right) \rightarrow C^{\infty}\left(\zeta^{0}\right)
$$

is equal to the Euler-Poincaré characteristic of $X$ with coefficients in the sheaf $\Omega(\xi)$, that is,

$$
\begin{aligned}
\chi(X, \Omega(\xi))= & \operatorname{dim}\left(\text { kernel of } d_{\bar{z}}+d_{\bar{z}^{*}}^{*}: C^{\infty}\left(\zeta^{e}\right) \rightarrow C^{\infty}\left(\zeta^{0}\right)\right) \\
& -\operatorname{codim}\left(\text { image of } d_{\bar{z}}+d_{\bar{z}^{*}}^{*}: C^{\infty}\left(\zeta^{e}\right) \rightarrow C^{\infty}\left(\zeta^{0}\right)\right) .
\end{aligned}
$$

The adjoint of the operator $d_{\bar{z}}+d_{\bar{z}}^{*}: C^{\infty}\left(\zeta^{e}\right) \rightarrow C^{\infty}\left(\zeta^{0}\right)$ is the operator $d_{\bar{z}}+d_{\bar{z}}^{*}: C^{\infty}\left(\zeta^{0}\right) \rightarrow C^{\infty}\left(\zeta^{e}\right)$ and we have

$$
\left(d_{\bar{z}}+d^{*_{\bar{z}}}\right)\left(d_{\bar{z}}+d^{*}\right)=d_{\bar{z}} d_{\bar{z}}+d_{\bar{z}}{ }_{\bar{z}} d_{\bar{z}}=-\Delta_{\bar{z}},
$$

$\Delta_{z}$ being the complex analogue of the Laplace-Beltrame operator. The operator $\Delta_{\bar{z}}$ is a self-adjoint elliptic operator from $C^{\infty}\left(\zeta^{q}\right) \rightarrow C^{\infty}\left(\zeta^{q}\right), 0 \leq q \leq n$.

Let $\lambda$ be a non-negative real number, and $S_{q}(\lambda)$ be the eigenspace of the operator $\Delta_{\bar{z}}: C^{\infty}\left(\zeta^{q}\right) \rightarrow C^{\infty}\left(\zeta^{q}\right)$ corresponding to $\lambda$. Then the following proposition is an immediate consequence of an argument due to Atiyah Bott; see $[4, \S 3]$. 


\section{Proposition 1.4.}

$$
\sum_{q=0}^{n}(-1)^{q} \operatorname{dim} S_{q}(\lambda)=\left\{\begin{array}{c}
0, \text { if } \lambda>0, \\
\text { the analytic index of the operator } \\
d_{\bar{z}}+d_{\bar{z}}^{*}: C^{\infty}\left(\zeta^{e}\right) \rightarrow C^{\infty}\left(\zeta^{0}\right), \text { if } \\
\lambda=0 .
\end{array}\right.
$$

In fact, for $\lambda>0, d_{\bar{z}}+d^{*}{ }_{z}$ induces an isomorphism of $\oplus S_{2 q}(\lambda) \rightarrow \oplus S_{2 q+1}(\lambda)$, and for $\lambda=0, \sum \operatorname{dim} S_{2 q}(\lambda)=$ dimension of the kernel of $d_{\bar{z}}+d_{{ }_{z}}^{*}: C^{\infty}\left(\zeta^{e}\right)$ $\rightarrow C^{\infty}\left(\zeta^{0}\right)$ and $\sum \operatorname{dim} S_{2 q+1}(\lambda)=$ dimension of the cokernel of $d_{\bar{z}}+d^{*}{ }_{i}$.

The operator $\Delta_{\bar{z}}: C^{\infty}\left(\zeta^{q}\right) \rightarrow C^{\infty}\left(\zeta^{q}\right)$ has an infinite sequence

$$
0 \geq \lambda_{1, q} \geq \lambda_{2, q} \geq \cdots \geq \lambda_{m, q} \geq \cdots \downarrow-\infty
$$

of eigenvalues, each eigenvalue being repeated as many times as its multiplicity indicates and corresponding sequence $\left\{\varphi_{m}\right\}$ of eigenforms forming a complete orthonormal set in the space $C^{\infty}\left(\zeta^{q}\right)$ with the hermitian inner product. Furthermore, the series

$$
e^{q}\left(t, z^{\prime}, z\right)=\sum \exp \left(\lambda_{m, q} l\right) \varphi_{m}\left(z^{\prime}\right) \otimes \varphi_{m}(z)
$$

converges uniformly on compact figures of $(0, \infty) \times X^{2}$ to the fundamental solution of the heat operator $\partial / \partial t-\Delta_{\bar{z}}$, and we have

$$
\left(\operatorname{Tr} e^{q}\right)(t, z, z)=\sum \exp \left(\lambda_{m, q} t\right)\left\langle\varphi_{m}(z), \varphi_{m}(z)\right\rangle,
$$

where $\langle$,$\rangle denotes the hermitian inner product in \zeta^{q}$, and $\operatorname{Tr}$ the trace of an endomorphism. Let

$$
(\operatorname{Tr} e)(t, z, z)=\sum_{q=0}^{n}(-1)^{q}\left(\operatorname{Tr} e^{q}\right)(t, z, z)
$$

Then

$$
\begin{aligned}
\int_{X}(\operatorname{Tr} \mathrm{e})(t, z, z) * 1= & \sum_{q=0}^{n}(-1)^{q} \sum_{m \geq 0} \exp \left(\lambda_{m, q} t\right), \quad t>0 \\
= & \text { the analytic index of the operator } d_{\bar{z}}+d_{z}^{*}: \\
& C^{\infty}\left(\zeta^{e}\right) \rightarrow C^{\infty}\left(\zeta^{0}\right) \text { by Proposition } 1.4 \\
= & \chi(X, \Omega(\xi)) \text { by Proposition } 1.3,
\end{aligned}
$$

$* 1$ being the volume element with respect to the hermitian metric in $T^{*}(X)$.

Thus we obtain the following theorem:

Theorem 1.5. Let $e^{q}\left(t, z^{\prime}, z\right)$ be the fundamental solution of the heat operator $\partial / \partial t-\Delta_{\bar{z}}$ acting on $(0, q)$-forms with values in the vector bundle $\xi$. Then we have the following integral expression for the Euler-Poincare characteristic $\chi(X, \Omega(\xi))$ : 


$$
\chi(X, \Omega(\xi))=\int_{X}\left(\sum_{q=0}^{n}(-1)^{q} \operatorname{Tr} e^{q}(t, z, z)\right) * 1, \quad t>0 .
$$

This theorem is of course well-known.

Moreover one can show that for any positive integer $N$ we have the following expansion

$$
\sum_{q=0}^{n}(-1)^{q} \operatorname{Tr} e^{q}(t, z, z)=t^{-n} \sum_{i=0}^{N} t^{i} f_{i}(z)+0\left(t^{N-n+1}\right), t \downarrow 0
$$

where $f_{0}, f_{1}, \cdots, f_{N}$ are $C^{\infty}$-functions defined on $X$.

In view of Theorem 1.5 and the above expansion, in order to express the Euler-Poincaré characteristic $\chi(X, \Omega(\xi))$ in terms of some topological invariants of $X$ and $\xi$, it is enough to do so for the element of $H^{2 n}(X, R)$ represented by $f_{n}(z) * 1$, and this is what we shall do in this paper. In fact, we shall prove the following theorem (under the assumption that the hemitian metric which we introduced in $T(X)$ is a Kaehler metric).

Theorem 1.6. Let $e^{q}\left(t, z^{\prime}, z\right)$ be the fundamental solution of the heat operator $\partial / \partial t-\Delta_{z}$ acting on $(0, q)$-forms with values in the vector bundle $\xi$. Then

$$
\sum_{q=0}^{n}(-1)^{q} \operatorname{Tr} e^{q}(t, z, z)=F(z)+0(t),
$$

where $F(z)$ is a $C^{\infty}$-function on $X$ such that the element of $H^{2 n}(X, R)$ represented by $F(z) * 1$ equals $[\mathrm{ch}(\xi) \mathscr{T}(X)]_{2 n}$.

Theorem 1.1 is of course an immediate consequence of Theorems 1.5 and 1.6.

$\S \S 2$ and 3 are devoted to some preliminaries. In $\S 4$ we outline the construction of the fundamental solution of the operator $\partial / \partial t-\Delta_{\bar{z}}$ acting on $(0, q)$-forms with values in the vector bundel $\xi$. In $\S 5$ we prove two crucial lemmas and in $\S 6$ we complete the proof of Theorem 1.6. The present paper is a natural outcome of the method developed in [3].

The author wishes to express his thanks to Professors M.S. Narasimhan and S. Ramanan for their interest in this work, and is also thankful to Professor C.P. Ramanujam for his help with Lemma 2.7.

\section{Algebraic preliminaries}

Let $V$ be a complex vector space, $n$ its complex dimension, $V^{*}$ the dual space of $V$, and $A$ a linear operator from $V$ into itself. Then for $1 \leq q \leq n$, there are two naturally defined linear operators $\wedge^{q} A$ (q-th exterior power of $A$ ) and $D^{q} A$ (derivation extension of $A$ ) from $\wedge^{q} V$ into itself such that 


$$
\begin{aligned}
\left(\wedge \wedge^{q} A\right)\left(v_{1} \wedge \cdots \wedge v_{q}\right) & =\left(A v_{1}\right) \wedge \cdots \wedge\left(A v_{q}\right) \\
\left(D^{q} A\right)\left(v_{1} \wedge \cdots \wedge v_{q}\right)= & \sum_{r=1}^{q} v_{1} \wedge \cdots \wedge v_{r-1} \wedge A\left(v_{r}\right) \wedge v_{r+1} \\
& \wedge \cdots \wedge v_{q}, \quad v_{1}, \cdots, v_{q} \in V .
\end{aligned}
$$

We define $\wedge^{0} A, D^{0} A$ respectively to be the identity endomorphism, zero endomorphism of the field of scalars, and denote the trace of a linear operator $B$ of $V$ into itself by $\operatorname{Tr} B$.

Lemma 2.1. Let $A_{1}, \cdots, A_{k}$ be linear operators from $V$ into itself $k \leq n$. Then

$$
\sum_{q=0}^{n}(-1)^{q} \operatorname{Tr}\left(D^{q} A_{1} \circ \cdots \circ D^{q} A_{k}\right)=\left\{\begin{array}{l}
0, \text { if } k<n, \\
(-1)^{n} \text { coefficient of } x_{1} \cdots x_{k} \text { in } \\
\operatorname{det}\left(x_{1} A_{1}+\cdots+x_{k} A_{k}\right) \text { if } k=n .
\end{array}\right.
$$

Proof (see Lemma 2.1 of [3]). Let $x_{1}, \cdots, x_{k}$ be $k$-parameters. Then we have

$$
\begin{aligned}
\operatorname{det}\left(I-e^{x_{1} A_{1}} \cdots e^{x_{k} A_{k}}\right) & =\sum_{q=0}^{n}(-1)^{q} \operatorname{Tr}\left(\wedge^{q}\left(e^{x_{1} A_{1}} \cdots e^{x_{k} A_{k}}\right)\right) \\
& =\sum_{q=0}^{n}(-1)^{q} \operatorname{Tr}\left(e^{x_{1} D^{q_{A_{1}}}} \cdots e^{x_{k} D^{\boldsymbol{q}_{A}}}\right) .
\end{aligned}
$$

Equating the coefficients of $x_{1} \cdots x_{k}$ in $\operatorname{det}\left(I-e^{x_{1} A_{1}} \cdots e^{x_{k} A_{k}}\right)$ and $\sum_{q=0}^{n}(-1)^{q}$ $\cdot \operatorname{Tr}\left(e^{x_{1} D^{q_{A_{1}}}} \ldots e^{x_{k} D^{q_{A k}}}\right)$, we get the result.

Let $V, W$ be complex vector spaces, and $n$ the dimension of $V$. For $0 \leq q \leq n$, let $\varphi_{q}: \operatorname{Hom}(W, W) \times \operatorname{Hom}(V, V) \rightarrow \operatorname{Hom}\left(W \otimes \wedge^{q} V, W \otimes \wedge^{q} V\right)$ be the map defined by

$$
\varphi_{q}(B, C)=B \otimes D^{q} C, \quad B \in \operatorname{Hom}(W, W), C \in \operatorname{Hom}(V, V) .
$$

The map $\varphi_{q}$ is bilinear and therefore defines a linear map $\tilde{\varphi}_{q}$ from $W \otimes W^{*}$ $\otimes V^{*} \otimes V(\approx \operatorname{Hom}(W, W) \otimes \operatorname{Hom}(V, V))$ to $\operatorname{Hom}\left(W \otimes \wedge^{q} V, W \otimes \wedge^{q} V\right)$. We shall denote the image of an element $A$ of $W \otimes W^{*} \otimes V^{*} \otimes V$ under $\tilde{\varphi}_{q}$ by $D^{q}(A)$.

Lemma 2.2. Let $A_{1}, \cdots, A_{k}$ be arbitrary elements of $W \otimes W^{*} \otimes V^{*} \otimes V$, $k<n$. Then

$$
\sum_{q=0}^{n}(-1)^{q} \operatorname{Tr}\left(D^{q} A_{1} \circ \cdots \circ D^{q} A_{k}\right)=0
$$

Proof. It is sufficient to prove the lemma when 


$$
A_{i}=B_{i} \otimes C_{i}, \quad B_{i} \in W \otimes W^{*}, \quad C_{i} \in V^{*} \otimes V, \quad 1 \leq i \leq k
$$

But then we have

$$
D^{q} A_{1} \circ \cdots \circ D^{q} A_{k}=\left(B_{1} \circ \cdots \circ B_{k}\right) \otimes\left(D^{q} C_{1} \circ \cdots \circ D^{q} C_{k}\right),
$$

and therefore

$$
\operatorname{Tr}\left(D^{q} A_{1} \circ \cdots \circ D^{q} A_{k}\right)=\operatorname{Tr}\left(B_{1} \circ \ldots \circ B_{k}\right) \operatorname{Tr}\left(D^{q} C_{1} \circ \ldots \circ D^{q} C_{k}\right),
$$

so that

$$
\begin{gathered}
\sum_{q=0}^{n}(-1)^{q} \operatorname{Tr}\left(D^{q} A_{1} \circ \cdots \circ D^{q} A_{k}\right)=\operatorname{Tr}\left(B_{1} \circ \cdots \circ B_{k}\right) \\
\cdot \sum_{q=0}^{n}(-1)^{q} \operatorname{Tr}\left(D^{q} C_{1} \circ \cdots \circ D^{q} C_{k}\right)=0 \text {, by Lemma } 2.1 .
\end{gathered}
$$

One can similarly prove the following lemma.

Lemma 2.3. Let $A_{1}, \cdots, A_{k}$ be arbitrary elements of $W \otimes W^{*} \otimes V^{*} \otimes V$, $k<n$, and $B_{1}, \cdots, B_{l}$ be arbitrary elements of $W \otimes W^{*}$. Let $\sigma$ be a permutation of $\{1, \cdots, k+l\}$, and for any integer $q$ between 0 and $n$ let the endomorphisms $S_{i}^{q}(1 \leq i \leq k+l)$ of $W \otimes \wedge^{q} V$ be defined by

$$
S_{\sigma(i)}^{q}= \begin{cases}D^{q} A_{i}, & \text { for } 1 \leq i \leq k, \\ B_{i-k} \otimes I_{q}, & \text { for } k+1 \leq i \leq k+l,\end{cases}
$$

where $I_{q}$ is the identity endomorphism of $\wedge^{q} V$. Then

$$
\sum_{q=0}^{n}(-1)^{q} \operatorname{Tr}\left(S_{1}^{q} \circ \cdots \circ S_{k+l}^{q}\right)=0 .
$$

Now let $V$ be a real vector space with a ' $J$-structure' (thus $J$ is a given linear operator from $V$ into itself such that $J^{2}=-1$ ). Suppose that we are given a positive definite symmetric bilinear form B in $V$ such that $B$ is invariant under $J$, that is,

$$
B(J x, J y)=B(x, y), \quad x, y \in V .
$$

Let $V^{*}$ be the dual space of $V$. Then the $J$-structure on $V$ induces canonically a $J$-structure on $V^{*}$ :

$$
\left\langle J X, Y^{*}\right\rangle=\left\langle X, J Y^{*}\right\rangle, \quad X \in V, Y^{*} \in V^{*} .
$$

Let $V^{c}, V^{* c}$ be the complexifications of $V$ and $V^{*}$, and put

$$
\begin{array}{cl}
V^{1,0}=\left\{v \in V^{c} \mid J v=i v\right\}, & V^{0,1}=\left\{v \in V^{c} \mid J v=-i v\right\}, \\
V^{* 1,0}=\left\{v \in V^{* c} \mid J v=i v\right\}, & V^{* 0,1}=\left\{v \in V^{* c} \mid J v=-i v\right\} .
\end{array}
$$


Then $V^{c}=V^{1,0} \oplus V^{0,1}, V^{* c}=V^{* 1,0} \oplus V^{* 0,1}$, and furthermore

$$
\begin{array}{ll}
V^{* 1,0}=\left\{v^{*} \in V^{* c} \mid\left\langle v^{*}, w\right\rangle=0\right. & \text { for } \left.w \in V^{0,1}\right\}, \\
V^{* 0,1}=\left\{v^{*} \in V^{* c} \mid\left\langle v^{*}, w\right\rangle=0\right. & \text { for } \left.w \in V^{1,0}\right\} .
\end{array}
$$

Thus $V^{* 1,0}$ and $V^{* 0,1}$ are respectively the dual space of $V^{1,0}$ and $V^{0,1}$.

Let $2 n$ be the (real) dimension of $V$. There is a unique element (volume element) $e \in \wedge^{2 n} V^{*}$ such that $B(e, e)=1$ and for any basis $e_{1}, J e_{1}, \cdots, e_{n}, J e_{n}$ of $V^{*}$,

$$
e=\alpha e_{1} \wedge J e_{1} \wedge \cdots \wedge e_{n} \wedge J e_{n}, \text { with a positive constant } \alpha .
$$

We extend the bilinear form $B$ to $V^{c}\left(V^{* c}\right)$ as follows:

$$
B\left(X+i Y, X^{\prime}+i Y^{\prime}\right)=B\left(X, X^{\prime}\right)+B\left(Y, Y^{\prime}\right)+i B\left(Y, X^{\prime}\right)-i B\left(X, Y^{\prime}\right) .
$$

The bilinear form induces a map $\phi^{\prime}$ of $V$ into $V^{*}$ :

$$
\left\langle\psi^{\prime}(x), y\right\rangle=B(x, y), \quad x, y \in V .
$$

We extend the map $\psi^{\prime}$ by complex linearity to a map $\phi$ of $V^{c}$ into $V^{* c}$. The map $\psi$ is an isomorphism and thus defines an isomorphism $\phi \otimes I_{d}: V^{c} \otimes V^{* c}$ $\rightarrow V^{* c} \otimes V^{* c}, I_{d}$ being the identity endomorphism of $V^{* c}$. Combining the isomorphism $\phi \otimes I_{d}$ with the canonical map from $V^{* c} \otimes V^{* c}$ to $V^{* c} \wedge V^{* c}$ $\left(v_{1} \otimes v_{2} \mapsto v_{1} \wedge v_{2}\right)$ we get a map, which we shall denote by $\varphi$, from $V^{c} \otimes V^{* c}$ to $V^{* c} \wedge V^{* c}$.

Lemma 2.4. Let $A_{1}, \cdots, A_{n} \in V^{0,1} \otimes V^{* 0,1}$ (thus each $A_{i}$ is an endomorphism of $\left.V^{* 0,1}\right)$. Then

$$
\left(\sum_{q=0}^{n}(-1)^{q} \operatorname{Tr}\left(D^{q} A_{1} \circ \cdots \circ D^{q} A_{n}\right)\right) e=(-i)^{n} \varphi\left(A_{1}\right) \wedge \cdots \wedge \varphi\left(A_{n}\right) .
$$

Proof. There exist vectors $e_{1}, \cdots, e_{n}$ in $V$ such that $e_{1}, J e_{1}, \ldots, e_{n}, J e_{n}$ form an orthonormal basis (see Proposition 1.8 of [2]). Let $e_{1}^{*},-J e_{1}^{*}, \cdots$, $e_{n}{ }^{*},-J e_{n}{ }^{*}$ be the dual basis for $V^{*}$, and put $v_{j}=\frac{1}{2}\left(e_{j}+i J e_{j}\right)$ and $v^{*}{ }_{j}=$ $e_{j}^{*}+i J e_{j}^{*}$. Then $v_{1}, \cdots, v_{n}$ and $v_{1}^{*}, \cdots, v_{n}^{*}$ are dual bases for $V^{0,1}$ and $V^{* 0,1}$.

Let $A_{i}=\sum a_{j k}^{i} v_{j} \otimes v_{k}^{*}$. Then we have

$$
\begin{aligned}
& \sum_{q=0}^{n}(-1)^{q} \operatorname{Tr}\left(D^{q} A_{1} \circ \cdots \circ D^{q} A_{n}\right) \\
& =(-1)^{n} \text { coefficient of } x_{1} \cdots x_{n} \text { in det }\left(x_{1} A_{1}+\cdots+x_{n} A_{n}\right), \\
& \quad \text { by Lemma } 2.1 \\
& =(-1)^{n} \text { coefficient of } x_{1} \cdots x_{n} \text { in } \sum_{\sigma} \epsilon_{\sigma} \prod_{j=1}^{n}\left(\sum_{i} x_{i} a_{j \sigma(j)}^{i}\right), \\
& \quad \epsilon_{\sigma} \text { denoting the sign of the permutation } \sigma \\
& =(-1)^{n} \sum_{\sigma} \epsilon_{\sigma} \sum_{\rho} a_{1 \sigma(1)}^{\rho(1)} \cdots a_{n \sigma(n)}^{\rho(n)} \\
& =(-1)^{n} \sum_{\sigma, \rho} \epsilon_{\sigma} \epsilon_{\rho} a_{\sigma(1) \rho(1)}^{1} \cdots a_{\sigma(n) \rho(n)}^{n},
\end{aligned}
$$




$$
\begin{aligned}
& \varphi\left(A_{1}\right) \wedge \cdots \wedge \varphi\left(A_{n}\right) \\
& =\frac{1}{2^{n}}\left(\sum a_{j k}^{1} \overline{v_{j}^{*}} \wedge v_{k}^{*}\right) \wedge \cdots \wedge\left(\sum a_{j k}^{n} \overline{v_{j}^{*}} \wedge v_{k}^{*}\right) \\
& =\frac{1}{2^{n}} \sum_{\sigma, \rho} a_{\sigma(1) \rho(1)}^{1} \cdots a_{\sigma(n) \rho(n)}^{n} \bar{v}_{\sigma(1)}^{*} \wedge v_{\rho(1)}^{*} \wedge \cdots \wedge \overline{v_{\sigma(n)}^{*}} \wedge v_{\rho(n)}^{*} \\
& =\frac{1}{2^{n}} \sum_{\sigma, \rho} \epsilon_{\sigma} \epsilon_{\rho} a_{\sigma(1) \rho(1)}^{1} \cdots a_{\sigma(n) \rho(n)}^{n} \bar{v}_{\sigma(1)}^{*} \wedge v_{\sigma(1)}^{*} \wedge \cdots \wedge \bar{v}_{\sigma(n)}^{*} \wedge v_{\sigma(n)}^{*} \\
& =\frac{1}{2^{n}} \sum_{\sigma, \rho} \epsilon_{\sigma} \epsilon_{\rho} a_{\sigma(1) \rho(1)}^{1} \cdots a_{\sigma(n) \rho(n)}^{n} \bar{v}_{1}^{*} \wedge v_{1}^{*} \wedge \cdots \wedge \bar{v}_{n}^{*} \wedge v_{n}^{*} \\
& =i^{n} \sum_{\sigma, \rho} \epsilon_{\sigma} \epsilon_{\rho} a_{\sigma(1) \rho(1)}^{1} \cdots a_{\sigma(n) \rho(n)}^{n} e_{1}^{*} \wedge J e_{1}^{*} \wedge \cdots \wedge e_{n}^{*} \wedge J e_{n}^{*} \\
& =i^{n}\left(\sum_{\sigma, \rho} \epsilon_{\sigma} \epsilon_{\rho} a_{\sigma(1) \rho(1)}^{1} \cdots a_{\sigma(n) \rho(n)}^{n}\right) e \\
& =(-i)^{n}\left(\sum_{q=0}^{n}(-1)^{q} \operatorname{Tr}\left(D^{q} A_{1} \circ \cdots \circ D^{q} A_{n}\right)\right) e .
\end{aligned}
$$

This completes the proof of lemma.

Now let $W$ be a complex vector space. There is a natural map $\varphi_{1}$ from $\left(W \otimes W^{*} \otimes V^{* c} \wedge V^{* c}\right) \times \cdots \times\left(W \otimes W^{*} \otimes V^{* c} \wedge V^{* c}\right)$ ( $k$-factors) into $W \otimes W^{*} \otimes \cdots \otimes W \otimes W^{*} \otimes \wedge^{2 k} V^{* c}$,

$$
\begin{aligned}
& \left(w_{1} \otimes w_{1}^{*} \otimes\left(v_{1} \wedge u_{1}\right), \cdots, w_{k} \otimes w_{k}^{*} \otimes\left(v_{k} \wedge u_{k}\right)\right) \\
& \mapsto w_{1} \otimes w_{1}^{*} \otimes \cdots \otimes w_{k} \otimes w_{k}^{*} \otimes\left(v_{1} \wedge u_{1} \wedge \cdots \wedge v_{k} \wedge u_{k}\right), \\
& w_{i} \in W, \quad w_{i}^{*} \in W^{*}, \quad v_{i}, u_{i} \in V^{* c}
\end{aligned}
$$

Moreover the map $\varphi_{2}$ from $\left(W \otimes W^{*}\right) \times \cdots \times\left(W \otimes W^{*}\right)$ ( $k$-factors) into $C$ defined by

$$
\varphi_{2}\left(f_{1}, \cdots, f_{k}\right)=\operatorname{Tr}\left(f_{1} \circ \cdots \circ f_{k}\right), \quad f_{i} \in W \otimes W^{*}(\approx \operatorname{Hom}(W, W))
$$

is bilinear and therefore defines a linear map $\tilde{\varphi}_{2}$ from $W \otimes W^{*} \otimes \cdots \otimes W \otimes$ $W^{*}$ to $C$. Let $\tilde{\varphi}=\left(\tilde{\varphi}_{2} \otimes I_{d}\right) 0 \varphi_{1}, I_{d}$ being the identity endomorphism of $\wedge^{2 k} V^{* c}$.

Lemma 2.5. Let $A_{1}, \cdots, A_{n}$ be elements of $W \otimes W^{*} \otimes V^{0,1} \otimes V^{* 0,1}$. Then

$$
\left(\sum_{q=0}^{n}(-1)^{q} \operatorname{Tr}\left(D^{q} A_{1} \circ \cdots \circ D^{q} A_{n}\right)\right) e=(-i)^{n} \tilde{\varphi}\left(\left(I_{d} \otimes \varphi\right) A_{1}, \cdots,\left(I_{d} \otimes \varphi\right) A_{n}\right) .
$$

Proof. It is sufficient to prove the lemma for $A_{i}=B_{i} \otimes C_{i}, B_{i} \in W \otimes$ $W^{*}, C_{i} \in V^{0,1} \otimes V^{* 0,1}, 1 \leq i \leq n$. But in this case the lemma is an immediate consequence of the previous lemma.

Corollary 2.6. Assume $S \in W \otimes W^{*} \otimes V^{0,1} \otimes V^{* 0,1}$, and let $A_{1}, \cdots, A_{k}$ be arbitrary elements of $V^{0,1} \otimes V^{* 0,1}, k \leq n$, and $\sigma$ be a permutation of 1 to $n$. 
Assume $B_{o(r)}=I_{d} \otimes A_{r}$ for $1 \leq r \leq k, I_{d}$ being the identity endomorphism of $W$, and $B_{\sigma(r)}=S$ for $k+1 \leq r \leq n$. Then

$$
\begin{aligned}
& \left(\sum_{q=0}^{n}(-1)^{q} \operatorname{Tr}\left(D^{q} B_{1} \circ \cdots \circ D^{q} B_{n}\right)\right) e \\
& \quad=\left[\sum_{q=0}^{n}(-1)^{q} \operatorname{Tr}\left(\left(D^{q} S \circ \cdots \circ D^{q} S\right) \circ\left(I_{d} \otimes\left(D^{q} A_{1} \circ \cdots \circ D^{q} A_{k}\right)\right)\right)\right] e \\
& \quad=(-i)^{n} \tilde{\varphi}\left(\left(I_{d} \otimes \varphi\right) S, \cdots,\left(I_{d} \otimes \varphi\right) S\right) \wedge \varphi\left(A_{1}\right) \wedge \cdots \wedge \varphi\left(A_{k}\right) .
\end{aligned}
$$

The last lemma of this section is about the polynomial functions defined on the Lie algebra $\mathscr{G} l(n, C)$ of the Lie group $G L(n, C)$ (the group of $n \times n$ invertible matrices with complex entries). Let $f_{0}, f_{1}, \cdots, f_{n}$ be the polynomial functions defined on $\mathscr{G} l(n, C)$ by

$$
\operatorname{det}\left(\lambda I_{n}-\frac{1}{2 \pi \sqrt{-1}} X\right)=\sum_{r=0}^{n} \lambda^{n-r} f_{r}(X), \quad X \in \mathscr{G} l(n, C) .
$$

With respect to the canonical basis for $\mathscr{G} l(n, C)$, we can represent every element $X$ of $\mathscr{G} l(n, C)$ by a $n \times n$ matrix $\left(X_{j}^{i}\right)$ and then have the following explicit formula for the functions $f_{0}, f_{1}, \cdots, f_{n}$ :

$$
f_{r}(X)=\frac{(-1)^{r}}{(2 \pi \sqrt{-1})^{r} r !} \sum X_{i_{\sigma(1)}}^{i_{1}} \cdots X_{i_{\sigma(r)}}^{i_{r}},
$$

where the sum runs over all ordered tuples $\left(i_{1}, \cdots, i_{r}\right)$ and the permutation $\sigma$ of $\{1, \cdots, r\}$.

Let $r \leq n$ be a positive integer, and $\delta$ a permutation of $\{1, \cdots, r\}$. Define a polynomial function $g_{\delta}$ on the Lie algebra $\mathscr{G} l(n, C)$ by

$$
g_{\delta}(X)=\sum_{1 \leq i_{1}, \cdots, i_{r} \leq n} X_{i_{\delta(1)}}^{i_{1}} \cdots X_{i_{\delta(r)}}^{i_{r}}
$$

We can define the polynomial function $g_{\delta}$ in an intrinsic way as follows. Let $h_{\delta}$ be the endomorphism of $\otimes^{r} C^{n}$ ( $r$-th tensor product of the complex vector space $C^{n}$ ) defined by

$$
h_{\hat{\delta}}\left(v_{1} \otimes \cdots \otimes v_{r}\right)=v_{\tilde{\delta}(1)} \otimes \cdots \otimes v_{\delta(r)} .
$$

Then $g_{\hat{\delta}}(X)=$ Trace $\left(h_{\hat{\delta}} \circ \otimes^{r} X\right), X \in \mathscr{G} l(n, C)$, and

$$
\begin{aligned}
g_{\delta}\left(Y^{-1} X Y\right) & =\operatorname{Trace}\left(h_{\delta} \circ \otimes^{r}\left(Y^{-1} X Y\right)\right) \\
& =\operatorname{Trace}\left(h_{\delta} \circ\left(\otimes^{r} Y^{-1}\right) \circ\left(\otimes^{r} X\right) \circ\left(\otimes^{r} Y\right)\right) \\
& =\operatorname{Trace}\left(\left(\otimes^{r} Y^{-1}\right) \circ h_{\delta} \circ\left(\otimes^{r} X\right) \circ\left(\otimes^{r} Y\right)\right) \\
& =\operatorname{Trace}\left(h_{\delta} \circ\left(\otimes^{r} X\right) \circ\left(\otimes^{r} Y\right) \circ\left(\otimes^{r} Y^{-1}\right)\right) \\
& =\operatorname{Trace}\left(h_{\delta} \circ \otimes^{r} X\right)=g_{\delta}(X), \quad X, Y \in \mathscr{G} l(n, C) .
\end{aligned}
$$


Therefore $g_{\delta}$ is invariant under the action of the Lie group $G L(n, C)$.

Lemma 2.7. There exists a unique polynomial $P_{\delta}\left(Y_{1}, \cdots, Y_{r}\right)$ in the variables $Y_{1}, \cdots, Y_{r}, P_{\delta}\left(Y_{1}, \cdots, Y_{r}\right)=\sum_{\alpha_{1}+\cdots+r_{r}=r} P_{\delta}^{\alpha} Y_{1}^{\alpha_{1}} \cdots Y_{r}^{\alpha_{r}}$, such that

$$
g_{\delta}(X)=P_{\delta}\left(f_{1}(X), \cdots, f_{r}(X)\right), \quad X \in \mathscr{G} l(n, C) .
$$

Proof. We shall first prove the lemma for diagonal matrices. A diagonal matrix $X$ with entries $X_{1}, \cdots, X_{n}$ on its diagonal can be identified with the tuple $\left(X_{1}, \cdots, X_{n}\right)$. The functions $f_{1}, \cdots, f_{n}$ are then constant multiples of elementary symmetric functions of $X_{1}, \cdots, X_{n}$. Moreover, the function $g_{\delta}$ is easily verified to be a symmetric function of $X_{1}, \cdots, X_{n}$. In fact, any invariant polynomial function (on $\mathscr{G} l(n, C)$ ) restricted to diagonal matrices is a symmetric function. Hence there exists a unique polynomial $P_{\delta}\left(Y_{1}, \ldots, Y_{r}\right)$ such that (2.4) holds for all diagonal matrices $X$. Since the functions $P_{\delta}, f_{1}, \cdots, f_{n}$ are invariant under the action of the group $G L(n, C)$, we have (2.4) for all matrices $X$ which can be diagonalized. In particular (2.4) holds for all matrices $X$ which have distinct eigenvalues. Since the matrices which have distinct eigenvalues form an open set and both sides of (2.4) are analytic functions, we have (2.4) for all matrices $X$.

\section{Commutation formulas for covariant differentiation}

Let $h$ be a hermitian inner product in the holomorphic vector bundle $\xi$. Then there is a unique connection (called the hermitian connection) in the principle bundle associated with $\xi$ such that the metric tensor is parallel and the connection form is of type $(1,0)$; see [2, Chapter IX, $\S 10]$. Let $U$ be an open subset of $X$ such that $U$ is holomorphic to an open subset of $C^{n}$ ! (we shall denote the coordinate functions by $z_{1}, \cdots, z_{n}$ ) and the bundle $\xi$ is trivilized over $U$. Let $s_{1}, \cdots, s_{k}(k=$ rank of $E$ ) be the holomorphic cross sections of $E$ defined on $U$, which are everywhere linearly independent. Let

$$
h_{a b}=h\left(s_{a}, s_{b}\right), \quad 1 \leq a, b \leq k,
$$

and $\left(h^{a b}\right)$ be the inverse matrix of $\left(h_{a b}\right)$ so that $\sum_{c} h^{a c} h_{c b}$ equals 0 if $a \neq b$ and equals 1 if $a=b$.

With respect to the hermitian connection we have the following formulas for covariant differentiation:

$$
\nabla_{\partial / \partial z_{\alpha}}\left(s_{a}\right)=\sum_{b} l_{\alpha a}^{b} s_{b}, \quad 1 \leq \alpha \leq n, 1 \leq a \leq k,
$$

where $l_{\alpha a}^{b}=\sum_{c} \frac{\partial h_{a c}}{\partial z_{\alpha}} h^{c b}$, and $\nabla_{\partial / \partial \bar{z}_{\alpha}}\left(s_{a}\right)=0$.

Let $S$ be the curvature tensor associated with the hermitian connection and set $\left(S\left(\partial / \partial z_{\alpha}, \partial / \partial \bar{z}_{\beta}\right)\right) s_{a}=\sum_{\alpha} S_{a \alpha \beta}^{b} S_{b}$. Then $S_{a \alpha \bar{\beta}}^{b}=-\partial l_{\alpha a}^{b} / \partial \bar{z}_{\beta}$. 
Let $H$ be a hermitian metric in the tangent bundle $T(X)$ of $X$. Thus for each $x \in X, H$ is a positive definite inner product in $T_{x}(X)$ such that $H(J X, Y)=$ $i H(X, Y)=-H(X, J Y)$. Let $g$ be the Riemannian metric in $T(X)$ defined by

$$
g(X, Y)=\text { Real part of } H(X, Y), \quad X, Y \in T_{x}(X), \quad x \in X .
$$

We extend $g$ to the complexified tangent bundle $T^{c}(X)$ as follows:

$$
g\left(X_{1}+i X_{2}, Y_{1}+i Y_{2}\right)=g\left(X_{1}, Y_{1}\right)+g\left(X_{2}, Y_{2}\right)+i g\left(X_{2}, Y_{1}\right)-i g\left(X_{1}, Y_{2}\right) .
$$

Let $g_{\alpha \beta}=g\left(\partial / \partial z_{\alpha}, \partial / \partial z_{\beta}\right)$ and $\left(g^{\alpha \beta}\right)$ be the inverse matrix of $\left(g_{\alpha \beta}\right)$.

We consider the hermitian connection in the principle bundle associated with holomorphic vector bundle $T(X)$. The principle bundle associated with $T(X)$ can be regarded as a real vector bundle (say $\zeta$ ) with structure group $G L(2 n, R)$, and the hermitian connection defines a connection in $\zeta$. We extend the covariant differentiation in $T(X)$ (regarded as a real vector bundle) given by this connection in $\zeta$ to $T^{c}(X)$ as follows:

$$
\nabla_{Y_{1}+i Y_{2}}\left(Z_{1}+i Z_{2}\right)=\nabla_{Y_{1}}\left(Z_{1}\right)-\nabla_{Y_{2}}\left(Z_{2}\right)+i \nabla_{Y_{1}}\left(Z_{2}\right)+i \nabla_{Y_{2}}\left(Z_{1}\right)
$$

$Y_{1}, Y_{2}, Z_{1}, Z_{2}$ being vector fields defined on an open subset of $X$. Then we have the following formulas for covariant differentiation:

$$
\nabla_{\partial / \partial z_{\alpha}}\left(\partial / \partial z_{\beta}\right)=\sum_{\gamma} \Gamma_{\alpha \beta}^{\gamma} \partial / \partial z_{r}, \quad \nabla_{\partial / \partial \bar{z}_{\alpha}}\left(\partial / \partial z_{\beta}\right)=0, \quad 1 \leq \alpha, \beta \leq n,
$$

where

$$
\Gamma_{\alpha \beta}^{\gamma}=\sum \frac{\partial g_{\beta \varepsilon}}{\partial z_{\alpha}} g^{\varepsilon \gamma}
$$

Let $K$ be the curvature tensor, and set

$$
\begin{aligned}
& \left(K\left(\partial / \partial z_{\alpha}, \partial / \partial \bar{z}_{\beta}\right)\right)\left(\partial / \partial z_{\gamma}\right)=\sum_{\delta} K_{\gamma \alpha \beta}^{\delta} \partial / \partial z_{\delta}, \\
& \left(K\left(\partial / \partial z_{\alpha}, \partial / \partial \bar{z}_{\beta}\right)\right)\left(\partial / \partial \bar{z}_{\gamma}\right)=\sum K_{\bar{\gamma} \alpha \bar{\beta}}^{\bar{\delta}} \partial / \partial \bar{z}_{\delta} .
\end{aligned}
$$

Then

$$
K_{\gamma \alpha \bar{\beta}}^{\delta}=-\frac{\partial \Gamma_{\alpha \gamma}^{\delta}}{\partial \bar{z}_{\beta}} \quad \text { and } \quad K_{\bar{\gamma} \alpha \bar{\beta}}^{\bar{\delta}}=\frac{\partial \bar{\Gamma}_{\beta \gamma}^{\delta}}{\partial z_{\alpha}},
$$

where $\bar{\Gamma}_{\beta \gamma}^{\delta}$ denotes the complex conjugate of $\Gamma_{\beta \gamma}^{\delta}$. From now on we shall assume that the metric $g$ is a Kaehler metric. The Kaehler property is equivalent to the following relation of summetry:

$$
\Gamma_{\alpha \beta}^{\gamma}=\Gamma_{\beta \alpha}^{\gamma}
$$

(that is the hermitian connection has no torsion). 
Let

$$
\varphi=\left(\sum_{\beta_{1}<\cdots<\beta_{q}} \varphi_{\bar{\beta}_{1} \cdots \bar{\beta}_{q}}^{a} d \bar{z}_{\beta_{1}} \wedge \cdots \wedge d \bar{z}_{\beta_{q}}\right) a
$$

be a $C^{\infty}-(0, q)$ form defined on $U$ with values in the vector boundle $\xi$ (thus $\left.\varphi \in C^{\infty}\left(U, \xi \otimes \Lambda^{q} T^{* 0,1}(X)\right)\right)$. Then we have the following formulas for covariant differentiation (with respect to canonical connection in $\xi \otimes \Lambda^{q} T^{* c}(X)$ ) :

$$
\begin{aligned}
& \left(\nabla_{\partial / \partial \bar{z}_{\alpha}} \varphi\right)_{\bar{\beta}_{1} \cdots \bar{\beta}_{q}}^{a}=\partial \varphi_{\bar{\beta}_{1} \ldots \bar{\beta} q}^{a} / \partial \bar{z}_{\alpha}+\sum l_{\alpha b}^{a} \varphi_{\beta_{1} \cdots \bar{\beta} q}^{b},
\end{aligned}
$$

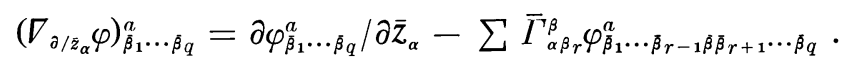

Let $A \in C^{\infty}\left(U, \xi \otimes \xi^{*} \otimes T^{0,1}(X) \otimes T^{* 0,1}(X)\right)$. Then for each $x \in U, A(x)$ is an element of $\xi_{x} \otimes \xi_{x}^{*} \otimes T_{x}^{0,1}(X) \otimes T_{x}^{* 0,1}(X)$ and hence defines an endomorphism $D^{q}(A(x))$ of $\xi_{x} \otimes \wedge^{q} T_{x}^{* 0,1}(X)$. Thus we get an endomorphism $D^{q} A$ of $C^{\infty}\left(U, \xi \otimes \wedge^{q} T^{* 0,1}(X)\right.$ ) (regarded as a module over the ring of $C^{\infty}$-complex valued functions on $U$ ):

$$
\left(D^{q} A(\alpha)\right)(x)=\left(D^{q} A(x)\right)(\alpha(x)), \quad \alpha \in C^{\infty}\left(U, \xi \otimes \wedge^{q} T^{* 0,1}(X)\right) .
$$

In the following lemmas covariant differentiations are taken with respect to the canonical connections in the bundles $\xi \otimes \xi^{*} \otimes T^{0,1}(X) \otimes T^{* 0,1}(X), \xi \otimes \wedge^{q}$ $T^{* 0,1}(X)$ induced by the hermitian connections in the bundles $\xi, T(X)$.

Lemma 3.1. Let $X_{1}, \cdots, X_{m}$ be $C^{\infty}$-vector fields defined on the open set $U, A \in C^{\infty}\left(U, \xi \otimes \xi^{*}\right)$, and the operators $\nabla_{X_{1}}, \cdots, \nabla_{X_{m}}$ of covariant differentiation be denoted respectively by $\nabla_{1}, \cdots, \nabla_{m}$. Then we have the following commutation relation;

$$
\begin{aligned}
& \nabla_{1} \circ \cdots \circ \nabla_{m} \circ\left(A \otimes I_{q}\right)=\left(A \otimes I_{q}\right) \circ \nabla_{1} \circ \cdots \circ \nabla_{m} \\
& \quad+\sum_{k=1}^{m} \sum_{\substack{\sigma(1)<\cdots<\sigma(k) \\
\sigma(k+1)<\cdots<\sigma(m)}}\left(\left(\nabla_{\sigma(1)} \circ \cdots \circ \nabla_{\sigma(k)}(A)\right) \otimes I_{q}\right) \circ \nabla_{\sigma(k+1)} \circ \cdots \circ \nabla_{\sigma(m)} .
\end{aligned}
$$

where $I_{q}$ denotes the identity endomorphism of $\wedge^{q} T^{* 0,1}(X)$.

Lemma 3.2. Let $X_{1}, \cdots, X_{m}$ be $C^{\infty}$-vector fields defined on the open set $U, A \in C^{\infty}\left(U, \xi \otimes \xi^{*} \otimes T^{0,1}(X) \otimes T^{* 0,1}(X)\right)$, and the operators $\nabla_{X_{1}}, \cdots, \nabla_{X_{m}}$ of covariant differentiation be denoted respectvely by $\nabla_{1}, \cdots, \nabla_{m}$. Then we have the following commutation relation;

$$
\begin{aligned}
& \nabla_{1} \circ \cdots \circ \nabla_{m} \circ D^{q} A=D^{q} A \circ \nabla_{1} \circ \cdots \circ \nabla_{m} \\
& \quad+\sum_{k=1}^{m} \sum_{\substack{\sigma(1)<\cdots<\sigma(k) \\
\sigma(k+1)<\cdots<\sigma(m)}} D^{q}\left(\nabla_{\sigma(1)} \circ \cdots \circ \nabla_{\sigma(k)}(A)\right) \circ \nabla_{\sigma(k+1)} \circ \cdots \circ \nabla_{\sigma(m)},
\end{aligned}
$$

where the second sum on the right hand side runs over all permutations $\sigma$ of $\{1, \cdots, m\}$ such that $\sigma(1)<\ldots<\sigma(k)$ and $\sigma(k+1)<\ldots<\sigma(m)$. 
The proofs of Lemmas 3.1 and 3.2 by induction on $m$ are analogous to the proofs of Lemmas 3.2 and 3.3 of [3]. Here we shall not go into the details of the proof which are quite straight forward.

For any two vector fields $X, Y$ defined on $U, K(X, Y)$ is an endomorphism of $C^{\infty}\left(U, T^{* c}(X)\right)$ and maps $C^{\infty}\left(U, T^{* 0,1}(X)\right)$ into itself. Therefore we can regard $K(X, Y)$ as an endomorphism of $C^{\infty}\left(U, T^{* 0,1}(X)\right)$, and then have

$$
\begin{aligned}
&\left(V_{X} \circ \nabla_{Y}-\nabla_{Y} \circ \nabla_{X}\right) \alpha=\left(D ^ { q } \left(I_{\xi} \otimes\right.\right.K(Y, X)) \\
&-\left.S(Y, X) \otimes I_{q}+\nabla_{[X, Y]}\right)(\alpha), \\
& \alpha \in C^{\infty}\left(U, \xi \otimes \wedge^{q} T^{* 0,1}(X)\right),
\end{aligned}
$$

$I_{\xi}$ denoting identity endomorphism of $\xi$ and $I_{q}$ the identity endomorphism of $\wedge^{q} T^{* 0,1}(X)$.

Formula (3.4) for $q=1$ is just the definition of curvature tensor. Moreover it is easy to see that if (3.4) is true for $\alpha=\varphi_{1}, \varphi_{2}$, where

$$
\varphi_{1} \in C^{\infty}\left(U, \xi \otimes \wedge^{q_{1}} T^{* 0,1}(X)\right), \quad \varphi_{2} \in C^{\infty}\left(U, \wedge^{q_{2}} T^{* 0,1}(X)\right),
$$

then it is true for $\alpha=\varphi_{1} \wedge \varphi_{2}$. Hence by induction on $q$, we get formula (3.4).

Lemma 3.3. Let $X, X_{1}, \cdots, X_{m}$ be $C^{\infty}$-vector fields defined on $U$, and the operators $\nabla_{X_{1}}, \cdots, \nabla_{X_{m}}$ of covariant differentiation be denoted respectively by $\nabla_{1}, \cdots, \nabla_{m}$. Then

$$
\begin{aligned}
& \nabla_{1} \circ \cdots \circ \nabla_{m} \circ \nabla_{X}=\nabla_{X} \circ \nabla_{1} \circ \cdots \circ \nabla_{m} \\
& +\sum_{j=0}^{m-1} \sum_{\substack{\sigma(1)<\cdots<\sigma(j+1) \\
\sigma(j+2)<\cdots<\sigma(m)}} D^{q}\left(I_{\xi} \otimes\left(\nabla_{\sigma(1)} \circ \cdots \circ \nabla_{\sigma(j)}\left(K\left(X, X_{\sigma(j+1)}\right)\right)\right)\right. \\
& \circ \nabla_{\sigma(j+2)} \circ \cdots \circ \nabla_{\sigma(m)} \\
& -\sum_{j=0}^{m-1} \sum_{\substack{\sigma(1)<\ldots<\sigma(j+1) \\
\sigma(j+2)<\cdots<\sigma(m)}}\left(\left(\nabla_{\sigma(1)} \circ \cdots \circ \nabla_{\sigma(j)}\left(S\left(X, X_{\sigma(j+1)}\right)\right)\right) \otimes I_{q}\right) \\
& \circ \nabla_{\sigma(j+2)} \circ \cdots \circ \nabla_{\sigma(m)} \\
& -\sum_{i=1}^{m} \nabla_{1} \circ \cdots \circ \nabla_{i-1} \circ \nabla_{\left[X_{i}, X\right]} \circ \nabla_{i+1} \circ \cdots \circ \nabla_{m} \text {. }
\end{aligned}
$$

Proof. Lemma 3.3 follows easily by arguing inductively on $m$ and using Lemma 3.1, Lemma 3.2 and formula (3.4).

\section{Construction of a parametrix and the fundamental solution}

We shall first obtain an expression for the operator $\Delta_{\bar{z}}$. This expression will be an analogoue of the expression (1) of $\S 3$ of [3]. Let $U$ be an open subset of $X$ such that $U$ is holomorphic to an open subset of $C^{n}$, and the bundle $\xi$ is trivilized over $U$. 
Let $\varphi=\left(\sum \varphi_{\beta_{1} \ldots \bar{\beta}_{q}}^{a} d \bar{z}_{\beta_{1}} \wedge \cdots \wedge d \bar{z}_{\beta_{q}}\right)_{a}$ be a $C^{\infty}(0, q)$ form defined on $U$ with values in the vector bundle $\xi$. We have the following expressions for the operators $d_{\tilde{z}}$ and $d_{z}^{*}$ (see [5, Chapter $\left.3, \S 10\right]$ ),

$$
\left(d_{\bar{z}} \varphi\right)_{\bar{\beta}_{1} \cdots \bar{\beta} q+1}^{a}=\sum(-1)^{r-1}\left(\nabla_{\bar{\beta}_{r}} \varphi\right)_{\bar{\beta}_{1} \ldots \hat{\beta}_{r} \cdots \bar{\beta} q+1}^{a},
$$

where " $\wedge$ " denotes that the particular term is to be omitted, and

$$
\left(d_{\bar{z}}^{*} \varphi\right)_{\bar{\beta}_{1} \cdots \bar{\beta}_{q-1}}^{a}=-\sum g^{\beta \alpha}\left(\nabla_{\alpha} \varphi\right)_{\bar{\beta} \bar{\beta}_{1} \cdots \bar{\beta} q-1}^{a} .
$$

Therefore

$$
\begin{aligned}
\left(d_{\bar{z}}^{*} d_{\bar{z}} \varphi\right)_{\bar{\beta}_{1} \cdots \bar{\beta} q}^{a}= & -\sum g^{\beta \alpha}\left(\nabla_{\alpha} \circ \nabla_{\bar{\beta}}(\varphi)\right)_{\bar{\beta}_{1} \cdots \bar{\beta} q}^{a} \\
& -\sum(-1)^{r} g^{\beta \alpha}\left(\nabla_{\alpha} \circ \nabla_{\bar{\beta} r}(\varphi)\right)_{\bar{\beta} \bar{\beta}_{1} \cdots \hat{\beta} \cdots \bar{\beta}_{q} q}^{a}, \\
\left(d_{\bar{z}} d_{\bar{z}}^{*} \varphi\right)_{\bar{\beta}_{1} \cdots \bar{\beta} q}^{a}= & -\sum(-1)^{r-1} g^{\beta \alpha}\left(\nabla_{\bar{\beta} r} \circ \nabla_{\alpha}(\varphi)\right)_{\bar{\beta} \bar{\beta}_{1} \cdots \hat{\beta}_{\hat{\beta}} \cdots \bar{\beta}_{\bar{\beta}} q}^{a} .
\end{aligned}
$$

Thus we get

$$
\Delta_{\bar{z}}=-\left(d^{*}{ }_{\bar{z}} d_{\bar{z}}+d_{\bar{z}} d_{\bar{z}}^{*}\right)=\sum g^{\beta \alpha} \nabla_{\alpha} \circ \nabla_{\bar{\beta}}(\varphi)+R_{q}(\varphi),
$$

where

$$
\begin{aligned}
& \left(R_{q}(\varphi)\right)_{\bar{\beta}_{1} \cdots \bar{\beta} q}^{a}=\sum(-1)^{r-1} g^{\beta \alpha}\left(\left(\nabla_{\bar{\beta}_{r}} \circ \nabla_{\alpha}-\nabla_{\alpha} \circ \nabla_{\bar{\beta}_{r}}\right) \varphi\right)_{\bar{\beta}_{\tilde{\beta}_{1}} \cdots \hat{\bar{\beta}}_{r} \cdots \bar{\beta}_{q} q}^{a} \\
& =\sum(-1)^{r-1} g^{\beta \alpha}\left\{\left(\partial / \partial \bar{z}_{\beta r}\right)\left(l_{\alpha b}^{a} \varphi_{\bar{\beta} \bar{\beta}_{1} \ldots \hat{\beta}_{r} \cdots \bar{\beta}_{q}}^{b}\right)\right.
\end{aligned}
$$

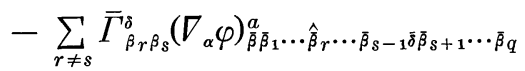

$$
\begin{aligned}
& -\sum \bar{\Gamma}_{\beta r \beta}^{\delta}\left(\nabla_{\alpha} \varphi\right)_{\bar{\delta}_{\bar{\beta}} \cdots \hat{\beta}_{r} \cdots \beta_{q}}^{a} \\
& +\left(\partial / \partial z_{\alpha}\right)\left(\sum_{r \neq s} \bar{\Gamma}_{\beta r \beta_{s}}^{\delta} \varphi_{\bar{\beta} \bar{\beta}_{1} \cdots \hat{\beta}_{r} \cdots \bar{\beta}_{s-1} \bar{\delta} \bar{\beta}_{s+1} \cdots \bar{\beta}_{\bar{\beta}} q}^{a}\right) \\
& \left.+\left(\partial / \partial z_{\alpha}\right)\left(\Gamma_{\beta_{r} \beta}^{\delta} \varphi_{\bar{\delta}_{\bar{\beta}} \cdots \hat{\beta}_{r} \cdots \bar{\beta}_{q}}^{\bar{a}}\right)-l_{\alpha b}^{a}\left(\nabla_{\bar{\beta}_{r}} \varphi\right)_{\bar{\beta} \bar{\beta}_{1} \cdots \hat{\beta}_{r} \ldots \bar{\beta}_{\beta}}^{b}\right\} .
\end{aligned}
$$

By the relation $\Gamma_{\beta \gamma}^{\alpha}=\Gamma_{\gamma \beta}^{\alpha}$, the second and fourth terms on the right side of the above equation are zero, and we thus obtain

$$
\begin{aligned}
& \left(R_{q}(\varphi)\right)_{\beta_{1} \cdots \bar{\beta} q}^{a}=\sum(-1)^{r-1} g^{\beta \alpha}\left\{\left(\partial l_{\alpha b}^{a} / \partial \bar{z}_{\beta_{r}}\right) \varphi_{\beta_{\beta_{1}} \cdots \hat{\beta}_{r} \cdots \bar{\beta} q}^{b}\right. \\
& -\bar{\Gamma}_{\beta r_{\beta} \beta}^{\delta} l_{\alpha b}^{a} \varphi_{\bar{\delta} \bar{\beta}_{1} \cdots \hat{\beta}_{r} \cdots \bar{\beta}_{q}}^{b}+\left(\partial \bar{\Gamma}_{\beta r_{\beta}}^{\delta} / \partial z_{\alpha}\right) \varphi_{\delta \bar{\beta}_{1} \cdots \hat{\beta}_{r} \cdots \bar{\beta}_{q}}^{a} \\
& +l_{\alpha b}^{a} \bar{\Gamma}_{\beta \gamma \beta}^{\delta} \varphi_{\bar{\delta}_{\bar{\beta}} \cdots \hat{\beta}_{r} \cdots \bar{\beta} q}^{b} \\
& \left.+l_{\alpha b}^{a} \sum_{r \neq s} \bar{\Gamma}_{\beta_{r} \beta_{s}}^{\delta} \varphi_{\beta \beta_{1} \cdots \hat{\beta}_{r} \bar{\beta}_{s-1} \bar{\delta} \bar{\beta}_{s+1} \cdots \bar{\beta}_{q}}^{b}\right\} \text {. }
\end{aligned}
$$

Therefore

$$
\begin{aligned}
& \left(R_{q}(\varphi)\right)_{\beta_{1} \cdots \beta_{q}}^{a}=\sum g^{\beta \alpha}\left(\partial l_{\alpha b}^{a} / \partial \bar{z}_{\beta r}\right) \varphi_{\beta_{1} \cdots \beta_{r-1} \beta \bar{\beta}_{r+1} \cdots \bar{\beta}_{q}}^{b}
\end{aligned}
$$

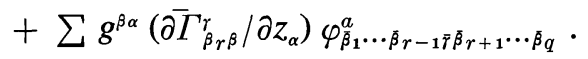


At each point $z \in X$, the curvature tensor fields $S$ and $K$ are elements of $\xi_{z} \otimes \xi_{z}^{*} \otimes T_{z}^{*}(X) \otimes T_{z}^{*}(X), T_{z}(X) \otimes T_{z}^{*}(X) \otimes T_{z}^{*}(X) \otimes T_{z}^{*}(X)$ respectively. But by the hermitian metric we can identify $T_{z}^{*}(X)$ with $T_{z}(X)$. Moreover there are projection operators from $T_{z}^{c}(X), T_{z}^{* c}(X)$ onto $T_{z}^{0,1}(X), T_{z}^{* 0,1}(X)$ respectively. By using the above isomorphism and the projection operators, $S$ and $K$ define elements (which we shall also denote by $S$ and $K$ ) of $\xi_{z} \otimes$ $\xi_{z}^{*} \otimes T_{z}^{0,1}(X) \otimes T_{z}^{* 0,1}(X), T_{z}^{0,1}(X) \otimes T_{z}^{* 0,1}(X) \otimes T_{z}^{0,1}(X) \otimes T_{z}^{* 0,1}(X)$. Thus we may have

$$
\begin{aligned}
& S=\sum S_{b \bar{\gamma}}^{a \tilde{\beta}} s_{a} \otimes s_{b} * \otimes \frac{\partial}{\partial \bar{z}_{\beta}} \otimes d \bar{z}_{r}, \\
& K=\sum K_{\bar{\beta} \overline{\bar{\delta}}}^{\alpha \bar{\gamma}} \frac{\partial}{\partial \bar{z}_{\alpha}} \otimes d \bar{z}_{\beta} \otimes \frac{\partial}{\partial \bar{z}_{r}} \otimes d \bar{z}_{\bar{\delta}}
\end{aligned}
$$

where

$$
S_{b \bar{\gamma}}^{a \bar{\beta}}=-\sum_{\alpha} g^{\beta \alpha} \frac{\partial l_{\alpha b}^{a}}{\partial \bar{z}_{\bar{\gamma}}}, \quad K_{\bar{\beta} \bar{\delta}}^{\alpha \bar{\gamma}}=\sum_{\varepsilon} g^{\gamma \varepsilon} \frac{\partial \bar{\Gamma}_{\delta \beta}^{\alpha}}{\partial z_{\varepsilon}} .
$$

By contracting the second and third indices the tensor field $K$ defines a tensor field $\tilde{K}$ of type $(1,1)$ :

$$
\tilde{K}=\sum_{\alpha, \beta, \gamma} K_{\bar{\beta} \bar{\gamma}}^{\bar{\alpha} \bar{\beta}} \frac{\partial}{\partial z_{\alpha}} \otimes d \bar{z}_{\gamma}
$$

The tensor fields $S$ and $\tilde{K}$, as we have stated in $\S 3$, define endomorphisms $D^{q} S, I_{\xi} \otimes D^{q} \tilde{K}$ of $C^{\infty}\left(X, \xi \otimes \wedge^{q} T^{* 0,1}(X)\right)$, and now we can write (4.2) as

$$
R_{q}(\varphi)=-D^{q} S(\varphi)+\left(I_{\xi} \otimes D^{q} \tilde{K}\right)(\varphi) .
$$

Hence by (4.1) we get the expression

$$
\Delta_{\bar{z}}=\sum g^{\beta \alpha} \nabla_{\alpha} \circ \nabla_{\bar{\beta}}+I_{\xi} \otimes D^{q} \tilde{K}-D^{q} S .
$$

Therefore for any complex-valued $C^{\infty}$-function $f$ defined on $U$ we have

$$
\begin{gathered}
\Delta_{\bar{z}}(f \varphi)=\left(\Delta_{\bar{z}} f\right) \varphi+f\left(\Delta_{\bar{z}} \varphi\right)+g^{\beta \alpha}\left(\nabla_{\bar{\beta}} f\right)\left(\nabla_{\alpha} \varphi\right)+g^{\beta \alpha}\left(\nabla_{\alpha} f\right)\left(\nabla_{\bar{\beta}} \varphi\right), \\
\Delta_{\bar{z}} f=g^{\beta \alpha} \frac{\partial^{2} f}{\partial z_{\alpha} \partial \bar{z}_{\beta}} .
\end{gathered}
$$

We now proceed to construct a parametrix for the fundamental solution of the heat operator $\partial / \partial t-\Delta_{\tilde{z}}$. We shall first fix some notation. By a double $(0, q)$-form $\varphi$ defined on an open subset $W$ of $X \times X$, we shall mean an element of $C^{\infty}\left(W,\left(\xi \otimes \wedge^{q} T^{* 0,1}(X)\right) \otimes\left(\xi \otimes \wedge^{q} T^{* 0,1}(X)\right)\right)$. Thus for each $\left(z^{\prime}, z\right) \in W$, 
we have $\varphi\left(z^{\prime}, z\right) \in \xi_{z^{\prime}} \otimes \wedge^{q} T_{z^{\prime}}^{* 0,1}(X) \otimes \xi_{z} \otimes \wedge^{q} T_{z}^{* 0,1}(X)$. Let $z \in X$ and $v \in \xi_{z} \otimes$ $\wedge^{q} T_{z}^{* 0,1}(X) \otimes \xi_{z} \otimes \wedge^{q} T_{z}^{* 0,1}(X)$. Hermitian metrices in $\xi$ and $T(X)$ introduce canonically a hermitian metric in $\xi \otimes \wedge^{q} T^{* 0,1}(X)$. By this hermitian metric in $\xi \otimes \wedge{ }^{q} T^{* 0,1}(X)$ we can identify $\xi_{z} \otimes \wedge^{q} T_{z}^{* 0,1}(X)$ with its dual vector space, and thus there is a canonical isomorphism of $\left(\xi_{z} \otimes \wedge^{q} T_{z}^{* 0,1}(X)\right) \otimes\left(\xi_{z} \otimes\right.$ $\left.\wedge^{q} T_{z}^{* 0,1}(X)\right)$ with $\left(\xi_{z} \otimes \wedge^{q} T_{z}^{* 0,1}(X)\right)^{*} \otimes\left(\xi_{z} \otimes \wedge^{q} T_{z}^{* 0,1}(X)\right)$. We therefore can regard $v$ as an endomorphism of $\xi_{z} \otimes \wedge^{q} T_{z}^{* 0,1}(X)$, and shall denote the trace of this endomorphism by $\operatorname{Tr} v$.

We fix an integer $q, 0 \leq q \leq n$ and construct a parametrix $H_{N}^{q}\left(t, z^{\prime}, z\right)$ in a sufficiently small neighbourhood of the diagonal in $X \times X$. We set

$$
H_{N}^{q}\left(t, z^{\prime}, z\right)=(2 \pi t)^{-n}\left(\exp \left(-r^{2} /(2 t)\right)\right) \sum_{i=0}^{N} t^{i} U^{i, q}\left(z^{\prime}, z\right),
$$

where $U^{i, q}\left(z^{\prime}, z\right)$ are double $(0, q)$-forms defined in a sufficiently small neighbourhood of the diagonal, and $r$ is the geodesic distance between $z^{\prime}$ and $z$. The forms $U^{i, q}\left(z^{\prime}, z\right)$ are to be determined such that $U^{0, q}\left(z^{\prime}, z^{\prime}\right)$ is the identity endomorphism of $\xi_{z^{\prime}} \otimes \wedge^{q} T_{z^{\prime}}^{* 0,1}(X)$ and

$$
\left(\frac{\partial}{\partial t}-\Delta_{\bar{z}}\right) H_{N}^{q}\left(t, z^{\prime}, z\right)=-(2 \pi t)^{-n} \exp \left(-r^{2} /(2 t)\right) t^{N} \Delta_{\bar{z}} U^{N, q}\left(z^{\prime}, z\right) .
$$

The integer $N$ is to be chosen larger than $n$, and these conditions determine the double forms $U^{i, q}\left(z^{\prime}, z\right)$ uniquely in a sufficiently small neighbourhood of the diagonal as we shall see now.

We have

$$
\Delta_{\bar{z}}\left(\exp \left(-\frac{r^{2}}{2 t}\right)\right)=g^{\beta \alpha} \frac{r^{2}}{t^{2}} \frac{\partial r}{\partial z_{\alpha}} \frac{\partial r}{\partial \bar{z}_{\beta}}-\frac{1}{2 t} \Delta_{\bar{z}}\left(r^{2}\right)
$$

But $g^{\beta \alpha} r^{2} \frac{\partial r}{\partial z_{\alpha}} \frac{\partial r}{\partial \bar{z}_{\beta}}=\frac{1}{2} r^{2}$; in fact, by denoting the Riemannian inner product by $\langle$,$\rangle we have$

$$
\begin{aligned}
g^{\beta \alpha} r^{2} \frac{\partial r}{\partial z_{\alpha}} \frac{\partial r}{\partial \bar{z}_{\beta}} & =\frac{1}{4}\left\langle d_{\bar{z}} r^{2}, d_{\bar{z}} r^{2}\right\rangle=\frac{1}{8}\left\{\left\langle d_{\bar{z}} r^{2}, d_{\bar{z}} r^{2}\right\rangle+\left\langle d_{z} r^{2}, d_{z} r^{2}\right\rangle\right\} \\
& =\frac{1}{8}\left\langle d r^{2}, d r^{2}\right\rangle=\frac{1}{2} r^{2}
\end{aligned}
$$

by choosing normal coordinates around $z^{\prime}$. Therefore we have

$$
\Delta_{\bar{z}}\left(\exp \left(-\frac{r^{2}}{2 t}\right)\right)=\left(\exp \left(-\frac{r^{2}}{2 t}\right)\right)\left(\frac{r^{2}}{2 t^{2}}-\frac{1}{2 t} \Delta_{\bar{z}} r^{2}\right)
$$

and, by (4.4), 


$$
\begin{aligned}
& \Delta_{\bar{z}}\left(\exp \left(-\frac{r^{2}}{2 t}\right) U^{i, q}\left(z^{\prime}, z\right)\right)=\exp \left(-\frac{r^{2}}{2 t}\right)\left\{\left(\frac{r^{2}}{2 t^{2}}-\frac{1}{2 t} \Delta_{\bar{z}}\left(r^{2}\right)\right) U^{i, q}\left(z^{\prime}, z\right)\right. \\
& \left.+\Delta_{\bar{z}} U^{i, q}\left(z^{\prime}, z\right)-g^{\beta \alpha} \frac{r}{t} \frac{\partial r}{\partial \bar{z}_{\beta}} \nabla_{\alpha} U^{i, q}\left(z^{\prime}, z\right)-g^{\beta \alpha} \frac{r}{t} \frac{\partial r}{\partial z_{\alpha}} \nabla_{\bar{\beta}} U^{i, q}\left(z^{\prime}, z\right)\right\}
\end{aligned}
$$

Therefore

$$
\begin{gathered}
\left(\frac{\partial}{\partial t}-\Delta_{\bar{z}}\right) H_{N}^{q}\left(t, z^{\prime}, z\right)=(2 \pi t)^{-n} \exp \left(-\frac{r^{2}}{2 t}\right) \\
\times \sum_{i=0}^{N} t^{i}\left\{\left(\frac{r^{2}}{2 t^{2}}+\frac{i-n}{t}-\frac{r^{2}}{2 t^{2}}+\frac{1}{2 t} \Delta_{\bar{z}}\left(r^{2}\right)\right) U^{i, q}\left(z^{\prime}, z\right)-\Delta_{\bar{z}} U^{i, q}\left(z^{\prime}, z\right)\right. \\
\left.+g^{\beta \alpha} \frac{r}{t} \frac{\partial r}{\partial \bar{z}_{\beta}} \nabla_{\alpha} U^{i, q}\left(z^{\prime}, z\right)+g^{\beta \alpha} \frac{r}{t} \frac{\partial r}{\partial z_{\alpha}} \nabla_{\bar{\beta}} U^{i, q}\left(z^{\prime}, z\right)\right\}
\end{gathered}
$$

Equating the coefficient of $\frac{t^{i-1}}{(2 \pi t)^{n}} \exp \left(-\frac{r^{2}}{2 t}\right)$ in $\left(\frac{\partial}{\partial t}-\Delta_{\bar{z}}\right) H_{N}^{q}\left(t, z^{\prime}, z\right)$ to
zero gives

$$
\begin{gathered}
\left(i-n+\frac{1}{2} \Delta_{\bar{z}}\left(r^{2}\right)\right) U^{i, q}\left(z^{\prime}, z\right)+\left(g^{\beta \alpha} r \frac{\partial r}{\partial \bar{z}_{\beta}} \nabla_{\alpha}+g^{\beta \alpha} r \frac{\partial r}{\partial z_{\alpha}} \nabla_{\beta}\right) U^{i, q}\left(z^{\prime} z\right) \\
=\Delta_{\bar{z}} U^{i-1, q}\left(z^{\prime}, z\right)
\end{gathered}
$$

On the other hand,

$$
r \frac{r}{d r}=g^{\beta \alpha} r \frac{\partial r}{\partial \bar{z}_{\beta}} \frac{\partial}{\partial z_{\alpha}}+g^{\beta \alpha} r \frac{\partial r}{\partial z_{\alpha}} \frac{\partial}{\partial \bar{z}_{\beta}},
$$

$d / d r$ denoting differentiation along the geodesic joining the points $z^{\prime}$ and $z$. In fact, we consider the differential form $\frac{1}{2} d r^{2}$ (defined in a sufficiently small neighbourhood of the point $z^{\prime}$ ), $d$ denoting the exterior differentiation with respect to the second variable. The Riemannian metric (which by definition is the real part of the hermitian metric) in $T(X)$ induces an isomorphism $\psi$ of $T_{x}(X)$ with $T_{x}^{*}(X)$ for all $x \in X$. By using normal coordinates one can easily see that $\psi^{-1}\left(\frac{1}{2} d r^{2}\right)=r \cdot d / d r$. On the other hand, we have

$$
\frac{1}{2} d r^{2}=\frac{1}{2} d_{\bar{z}} r^{2}+\frac{1}{2} d_{z} r^{2}=r \frac{\partial r}{\partial z_{\alpha}} d z_{\alpha}+r \frac{\partial r}{\partial \bar{z}_{\alpha}} d \bar{z}_{\alpha},
$$

so that

$$
\phi^{-1}\left(\frac{1}{2} d r^{2}\right)=r g^{\beta \alpha} \frac{\partial r}{\partial z_{\alpha}} \frac{\partial}{\partial \bar{z}_{\beta}}+r \frac{\partial r}{\partial \bar{z}_{\alpha}} g^{\alpha \beta} \frac{\partial}{\partial z_{\beta}} .
$$

Hence we obtain (4.8) and can write equation (4.7) as 


$$
\begin{array}{r}
\nabla_{r \cdot d / d r} U^{i, q}\left(z^{\prime} z\right)+\left(i-n+\frac{1}{2} \Delta_{\bar{z}}\left(r^{2}\right)\right) U^{i, q}\left(z^{\prime}, z\right)=\Delta_{\bar{z}} U^{i-1, q}\left(z^{\prime}, z\right), \\
0 \leq i \leq N .
\end{array}
$$

In [3] it is shown that the system of equations (4.3) of that paper has a unique solution in a neighbourhood of the diagonal. One can in an exactly similar way show that the system of equations (4.9) has a unique solution in a sufficiently small neighbourhood of the diagonal in $X \times X$, with the initial condition $U^{0, q}\left(z^{\prime}, z^{\prime}\right)$ equal to the identity endomorphism of $\xi_{z^{\prime}} \otimes \wedge^{q} T_{z^{\prime}}^{* 0,1}(X)$. Thus we can construct a parametrix $H_{N}^{q}\left(t, z^{\prime}, z\right)$ in a sufficiently small neighbourhood of the diagonal $W$ in $X \times X$. Starting with this parametrix $H_{N}^{q}\left(t, z^{\prime}, z\right)$ one can carry out the construction of the fundamental solution $e^{q}\left(t, z^{\prime}, z\right)$ for the heat operator $\partial / \partial t-\Delta_{\bar{z}}$. The method is completely analogous to the method used in $[3, \S 4]$. Furthermore one can show that

$$
\begin{aligned}
\left(\operatorname{Tr} e^{q}\right)\left(t, z^{\prime}, z^{\prime}\right) & =\left(\operatorname{Tr} H_{N}^{q}\right)\left(t, z^{\prime}, z^{\prime}\right)+0\left(t^{N-n+1}\right) \\
& =(2 \pi t)^{-n} \sum_{i=0}^{N} t^{i} \operatorname{Tr} U^{i, q}\left(z^{\prime}, z^{\prime}\right)+0\left(t^{N-n+1}\right), \text { by }(6) .
\end{aligned}
$$

(See the proof of formula (4.8) of [3].) Since $U^{0, q}\left(z^{\prime}, z^{\prime}\right)$ is the identity endomorphism of $\xi_{z^{\prime}} \otimes \wedge^{q} T_{z^{\prime}}^{* 0,1}(X)$, we get

$$
\begin{aligned}
\left(\operatorname{Tr} e^{q}\right)\left(t, z^{\prime}, z^{\prime}\right)=(2 \pi t)^{-n} & \left\{k\left(\begin{array}{l}
n \\
q
\end{array}\right)+t \operatorname{Tr} U^{1, q}\left(z^{\prime}, z^{\prime}\right)+\cdots\right. \\
& \left.+t^{N} \operatorname{Tr} U^{N, q}\left(z^{\prime}, z^{\prime}\right)\right\}+0\left(t^{N-n+1}\right), \quad t \downarrow 0,
\end{aligned}
$$

$k$ being the rank of the vector bundle $\xi$.

\section{Two crucial lemmas}

We fix a point $z^{\prime}$ of $X$, and let $U$ be an open neighbourhood of $z^{\prime}$ such that $U$ is holomorphic to an open subset of $C^{n}, \xi$ is trivilized over $U$ and any two points in $U$ can be joined by a unique geodesic lying in $U$. To start with some elementary observations let $X$ be a $C^{\infty}$-vector field defined on $U$. Then $\left[X, r \frac{d}{d r}\right]=X+Y$, where $Y$ is a $C^{\infty}$-vector field such that $Y\left(z^{\prime}\right)=0$. To prove this we introduce normal coordinates $\left(y_{1}, \cdots, y_{2 n}\right)$ in $U$ such that $z^{\prime}$ has coordinates $(0, \cdots, 0)$ and the matrix $\left(g_{i j}\left(z^{\prime}\right)\right)_{1 \leq i, j \leq 2 n}, g_{i j}=\left\langle\partial / \partial y_{i}, \partial / \partial y_{j}\right\rangle$, equals the identity matrix. Then $r \frac{d}{d r}=\sum y_{j} \partial / \partial y_{j}$, and if $X=\sum X_{j} \partial / \partial y_{j}$ for any $C^{\infty}$-function $f$ defined on $U$ we have

$$
\left(X \circ r \frac{d}{d r}\right)(f)=\sum X_{j} \frac{\partial f}{\partial y_{j}}+\sum X_{j} y_{k} \frac{\partial^{2} f}{\partial y_{j} \partial y_{k}},
$$




$$
\left(r \frac{d}{d r} \circ X\right)=\sum y_{j} \frac{\partial X_{k}}{\partial y_{j}} \frac{\partial f}{\partial y_{k}}+\sum y_{j} X_{k} \frac{\partial^{2} f}{\partial y_{j} \partial y_{k}}
$$

Therefore

$$
\left[X, r \frac{d}{d r}\right]=\sum X_{j} \frac{\partial}{\partial y_{j}}+\sum y_{j} \frac{\partial X_{k}}{\partial y_{j}} \frac{\partial}{\partial y_{k}}=X+Y
$$

where $Y$ is a $C^{\infty}$-vector field such that $Y\left(z^{\prime}\right)=0$. We also observe that $\left(\Delta_{\bar{z}}\left(r\left(z^{\prime}, z\right)\right)^{2}\right)\left(z^{\prime}, z^{\prime}\right)=2 n$, which follows from the relations $\Delta_{\bar{z}}=\frac{1}{2} \Delta$ and $\left(\Delta_{y}(r(x, y))^{2}\right)(x, x)=4 n, \Delta_{y}$ denoting the usual Laplace operator; the latter can be shown by using normal coordinates.

We now state our first lemma of this section, which we think can legitimately be named as a cancellation lemma.

Cancellation Lemma 5.1. Let $l_{1}, l_{2}, l_{3}, i$ be non-negative integers such that $l_{1} / 2+l_{2}+i<n$. Let $X_{1}, \cdots, X_{l_{1}}$ be $C^{\infty}$-vector fields defined on $U, A_{1}, \cdots, A_{l_{2}}$ be $C^{\infty}$-sections of $\xi \otimes \xi^{*} \otimes T^{0,1}(X) \otimes T^{* 0,1}(X)$ defined over $U$ and $B_{1}, \cdots, B_{l_{3}}$ be arbitrary elements of $C^{\infty}\left(U, \xi \otimes \xi^{*}\right)$. Let $\sigma$ be a permutation of $\left\{1, \ldots, l_{2}+l_{3}\right\}$. For any integer $q, 0 \leq q \leq n$, define the endomorphisms $S_{i}^{q}, 1 \leq i \leq l_{2}+l_{3}$, of $C^{\infty}\left(U, \xi \otimes \wedge^{q} T^{* 0,1}(X)\right)$ by

$$
S_{\sigma(i)}^{q}=\left\{\begin{array}{l}
D^{q}\left(A_{i}\right), \text { for } 1 \leq i \leq l_{2}, \\
B_{i-l_{2}} \otimes I_{q}, \text { for } l_{2}<i \leq l_{2}+l_{3}, I_{q} \text { being the identity endomorphism } \\
\text { of } \wedge^{q} T^{* 0,1}(X) .
\end{array}\right.
$$

Let the operators $\nabla_{X_{1}}, \cdots, \nabla_{X_{l_{1}}}$ be denoted by $\nabla_{1}, \cdots, \nabla_{l_{1}}$. Then we have

$$
\sum_{q=0}^{n}(-1)^{q} \operatorname{Tr}\left[S_{1}^{q} \circ \cdots \circ S_{l_{2}+l_{3}}^{q} \circ \nabla_{1} \circ \cdots \circ \nabla_{l_{1}}\left(U^{i, q}\left(z^{\prime}, z\right)\right)\right]\left(z^{\prime}, z^{\prime}\right)=0
$$

(all the operators act with respect to the variable $z$ ).

Proof. We shall prove the lemma by induction on $i$ and $l_{1}$. Let $j$ be a nonnegative integer and suppose that the lemma has been proved whenever $i<j$. We shall prove the lemma for $i=j$. Let $\mu_{q}$ be the operator defined by

$$
\mu_{q}=S_{1}^{q} \circ \cdots \circ S_{l_{2}+l_{3}}^{q} \circ \nabla_{1} \circ \cdots \nabla_{l_{1}} .
$$

First suppose that $l_{1}=0$. If $j=0$, then the lemma follows from Lemma 2.3. Therefore we can assume that $j>0$. The double form $U^{j, q}\left(z^{\prime}, z\right)$ satisfies the differential equation

$$
\begin{aligned}
\nabla_{r \frac{d}{d r}} & \left(U^{j, q}\left(z^{\prime}, z\right)\right)+\left(j-n+\frac{1}{2} \Delta_{\bar{z}}\left(r^{2}\right)\right) U^{j, q}\left(z^{\prime}, z\right)=\Delta_{\tilde{z}} U^{j-1, q}\left(z^{\prime}, z\right) \\
= & g^{\beta \alpha} \nabla_{\alpha} \nabla_{\hat{\beta}} U^{j-1, q}\left(z^{\prime}, z\right)+D^{q}\left(I_{\xi} \otimes \tilde{K}\right)\left(U^{j-1, q}\left(z^{\prime}, z\right)\right) \\
& -D^{q} S\left(U^{j-1, q}\left(z^{\prime}, z\right)\right) \quad \text { by }(4.3)
\end{aligned}
$$


Since the double form $\nabla_{r \frac{d}{d r}} U^{j, q}\left(z^{\prime}, z\right)$ is zero at $\left(z^{\prime}, z^{\prime}\right)$ we have

$$
\mu_{q}\left[\nabla_{r \frac{d}{d r}} U^{j, q}\left(z^{\prime}, z\right)\right]\left(z^{\prime}, z^{\prime}\right)=0 .
$$

Therefore applying the operator $\mu_{q}$ to both sides of equation (5.1) and then taking the trace we obtain

$$
\begin{aligned}
j \sum_{q=0}^{n}( & -1)^{q} \operatorname{Tr}\left[\mu_{q}\left(U^{j, q}\left(z^{\prime}, z\right)\right)\right]\left(z^{\prime}, z^{\prime}\right) \\
= & g^{\beta \alpha} \sum_{q=0}^{n}(-1)^{q} \operatorname{Tr}\left[\mu_{q} \circ \nabla_{\alpha} \circ \nabla_{\bar{\beta}}\left(U^{j-1, q}\left(z^{\prime}, z\right)\right)\right]\left(z^{\prime}, z^{\prime}\right) \\
& +\sum_{q=0}^{n}(-1)^{q} \operatorname{Tr}\left[\mu_{q} \circ D^{q}\left(I_{\xi} \otimes \tilde{K}\right)\left(U^{j-1, q}\left(z^{\prime}, z\right)\right)\right]\left(z^{\prime}, z^{\prime}\right) \\
& -\sum_{q=0}^{n}(-1)^{q} \operatorname{Tr}\left[\mu_{q} \circ D^{q}(S)\left(U^{j-1, q}\left(z^{\prime}, z\right)\right)\right]\left(z^{\prime}, z^{\prime}\right) .
\end{aligned}
$$

Since by the induction hypothesis each term on the right hand side of the above equation is zero, we have

$$
\sum_{q=0}^{n}(-1)^{q} \operatorname{Tr}\left[\mu_{q}\left(U^{j, q}\left(z^{\prime}, z\right)\right)\right]\left(z^{\prime}, z^{\prime}\right)=0 .
$$

Now suppose that $l_{1}>0$ and the lemma has been proved for smaller values of $l_{1}$. We wish to apply the operator $\mu_{q}$ to both sides of equation (5.1) and then take the trace. Let

$$
\begin{aligned}
& T_{1}^{q}=S_{1}^{q} \circ \cdots \circ S_{l_{2}+l_{3}}^{q} \circ \nabla_{r \frac{d}{d r}} \circ \nabla_{1} \circ \cdots \circ \nabla_{l_{1}}, \\
& T_{2}^{q}=\sum_{\sigma(2)<\cdots<\sigma\left(l_{1}\right)} S_{1}^{q} \circ \cdots \circ S_{l_{2}+l_{3}}^{q} \circ D^{q}\left(I_{\xi} \otimes K\left(r \frac{d}{d r}, X_{\sigma(1)}\right)\right) \\
& \quad \circ \nabla_{\sigma(2)} \circ \cdots \circ \nabla_{\sigma\left(l_{1}\right)}, \\
& T_{3}^{q}=\sum_{i=1}^{l_{1}} S_{1}^{q} \circ \cdots \circ S_{l_{2}+l_{3}}^{q} \circ \nabla_{1} \circ \cdots \circ \nabla_{i-1} \circ \nabla_{\left[x_{i}, r \frac{d}{d r}\right]} \circ \nabla_{i+1} \circ \cdots \circ \nabla_{l_{1}} .
\end{aligned}
$$

By Lemma 3.3, $\mu_{q} \circ \nabla_{r \frac{d}{d r}}=T_{1}^{q}+T_{2}^{q}+T_{3}^{q}+$ a sum of operators for each of which the induction hypothesis for $l_{1}$ applies. Since the vector field $r \frac{d}{d r}$ is zero
at the point $z^{\prime}$,

$$
\left[T_{1}^{q}\left(U^{j, q}\left(z^{\prime}, z\right)\right)\right]\left(z^{\prime}, z^{\prime}\right)=\left[T_{2}^{q}\left(U^{j, q}\left(z^{\prime}, z\right)\right)\right]\left(z^{\prime}, z^{\prime}\right)=0 .
$$

Also by the remark made at the beginning of this section,

$$
\begin{aligned}
& \sum_{i=1}^{l_{1}} S_{1}^{q} \circ \cdots \circ S_{l_{2}+l_{3}}^{q} \circ \nabla_{1} \circ \cdots \circ \nabla_{i-1} \circ \nabla_{\left[x_{i}, r \frac{d}{d r}\right]} \circ \nabla_{i+1} \circ \cdots \circ \nabla_{l_{1}} \\
& \quad=l_{1} \mu_{q}+\sum_{i=1}^{l_{1}} S_{1}^{q} \circ \cdots \circ S_{l_{2}+l_{3}}^{q} \circ \nabla_{1} \circ \cdots \circ \nabla_{i-1} \circ \nabla_{\tilde{X}_{i}} \circ \nabla_{i+1} \circ \cdots \circ \nabla_{l_{1}},
\end{aligned}
$$


where $\bar{X}_{i}$ 's are vector fields such that $\bar{X}_{i}\left(z^{\prime}\right)=0$. By Lemma 3.3,

$$
\begin{aligned}
\sum_{i=1}^{l_{1}} S_{1}^{q} \circ \cdots \circ S_{l_{2}+l_{3}}^{q} \circ \nabla_{1} \circ \cdots \circ \nabla_{i-1} \circ \nabla_{\tilde{X}_{i}} \circ \nabla_{i+1} \circ \cdots \circ \nabla_{l_{1}} \\
=\sum_{i=1}^{l_{1}} S_{1}^{q} \circ \cdots \circ S_{l_{2}+l_{3}}^{q} \circ \nabla_{\tilde{X}_{i}} \circ \nabla_{1} \circ \cdots \circ \hat{\nabla}_{i} \circ \cdots \circ \nabla_{l_{1}}+\text { a sum of } \\
\\
\quad \text { operatars for each of which the induction hypothesis for } l_{1} \\
\quad \text { applies. }
\end{aligned}
$$

Lastly since $\bar{X}_{i}\left(z^{\prime}\right)=0$ for $1 \leq i \leq l_{1}$, we have

$$
\left[\sum_{i=1}^{l_{1}} S_{1}^{q} \circ \cdots \circ S_{l_{2}+l_{3}}^{q} \circ \nabla_{\tilde{x}_{i}} \circ \nabla_{1} \circ \cdots \circ \hat{\nabla}_{i} \circ \cdots \circ \nabla_{l_{1}}\left(U^{j, q}\left(z^{\prime}, z\right)\right)\right]\left(z^{\prime}, z^{\prime}\right)=0 .
$$

Therefore the induction hypothesis gives

$$
\begin{aligned}
& \sum_{q=0}^{n}(-1)^{q} \operatorname{Tr}\left[\mu_{q} \circ \nabla_{r \frac{d}{d r}}\left(U^{j, q}\left(z^{\prime}, z\right)\right)\right]\left(z^{\prime}, z^{\prime}\right) \\
& \quad=l_{1} \sum_{q=0}^{n}(-1)^{q} \operatorname{Tr}\left[\mu_{q}\left(U^{j, q}\left(z^{\prime}, z\right)\right)\right]\left(z^{\prime}, z^{\prime}\right),
\end{aligned}
$$

and also

$$
\begin{gathered}
\mu_{q}\left(\Delta_{\bar{z}}\left(r^{2}\right) U^{j, q}\left(z^{\prime}, z\right)\right)=\Delta_{\bar{z}}\left(r^{2}\right) \mu_{q}\left(U^{j, q}\left(z^{\prime}, z\right)\right) \\
+\sum_{\substack{k=1 \\
l_{1}}} \sum_{\substack{\sigma(1)<\cdots<\sigma(k) \\
\sigma(k+1)<\cdots<\sigma\left(l_{1}\right)}}\left(\nabla_{\sigma(1)} \circ \cdots \circ \nabla_{\sigma(k)}\left(\Delta_{\bar{z}}\left(r^{2}\right)\right)\right) S_{1}^{q} \circ \cdots \circ S_{l_{2}+l_{3}}^{q} \\
\circ \nabla_{\sigma(k+1)} \circ \cdots \circ \nabla_{\sigma\left(l_{1}\right)}\left(U^{j, q}\left(z^{\prime}, z\right)\right) .
\end{gathered}
$$

Thus by the induction hypothesis for $l_{1}$ we obtain

$$
\begin{aligned}
& \sum_{q=0}^{n}(-1)^{q} \operatorname{Tr}\left[\mu_{q}\left(\Delta_{\bar{z}}\left(r^{2}\right) U^{j, q}\left(z^{\prime}, z\right)\right)\right]\left(z^{\prime}, z^{\prime}\right) \\
& \quad=2 n \sum_{q=0}^{n}(-1)^{q} \operatorname{Tr}\left[\mu_{q}\left(U^{j, q}\left(z^{\prime}, z\right)\right)\right]\left(z^{\prime}, z^{\prime}\right) .
\end{aligned}
$$

Similarly, by the induction hypothesis (for $i$ ) we have

$$
\sum_{q=0}^{n}(-1)^{q} \operatorname{Tr}\left[\mu_{q} \circ g^{\beta \alpha} \nabla_{\alpha} \circ \nabla_{\bar{\beta}}\left(U^{j-1, q}\left(z^{\prime}, z\right)\right)\right]\left(z^{\prime}, z^{\prime}\right)=0 .
$$

Lastly Lemma 3.2 together with the induction hypothesis (for $i$ ) gives

$$
\begin{gathered}
\sum_{q=0}^{n}(-1)^{q} \operatorname{Tr}\left[\mu_{q} \circ D^{q}\left(I_{\xi} \otimes \tilde{K}\right)\left(U^{j-1, q}\left(z^{\prime}, z\right)\right)\right]\left(z^{\prime}, z^{\prime}\right)=0, \\
\sum_{q=0}^{n}(-1)^{q} \operatorname{Tr}\left[\mu_{q} \circ D^{q} S\left(U^{j-1, q}\left(z^{\prime}, z\right)\right)\right]\left(z^{\prime}, z^{\prime}\right)=0 .
\end{gathered}
$$


Therefore applying the operator $\mu_{q}$ to both sides of equation (5.1) and then taking the trace we get

$$
\left(l_{1}+j\right) \sum_{q=0}^{n}(-1)^{q} \operatorname{Tr}\left[\mu_{q}\left(U^{j, q}\left(z^{\prime}, z\right)\right)\right]\left(z^{\prime}, z^{\prime}\right)=0 .
$$

Since $l_{1}+j>0$, we have

$$
\sum_{q=0}^{n}(-1)^{q} \operatorname{Tr}\left[\mu_{q}\left(U^{j, q}\left(z^{\prime}, z\right)\right)\right]\left(z^{\prime}, z^{\prime}\right)=0,
$$

which completes the proof of Lemma 5.1.

We now come to our next lemma of this section, and shall first introduce some notation. Greek letters $\alpha, \beta, \ldots$ will run from 1 to $n$. We shall denote the operators $\nabla_{\partial / \partial z_{\alpha}}, V_{\partial / \partial \bar{z}_{\alpha}}$ by $\nabla_{\alpha}, \nabla_{\bar{\alpha}}$ respectively, and the element $K\left(\partial / \partial z_{\alpha}, \partial / \partial \bar{z}_{\beta}\right)$ of $T_{z}^{0,1}(X) \otimes T_{z}^{* 0,1}(X)$ by $K(\alpha, \bar{\beta})\left(K\left(\partial / \partial z_{\alpha}, \partial / \partial \bar{z}_{\beta}\right)\right.$ is in fact an endomorphism of $T_{z}^{c}(X)$ and it maps $T_{z}^{* 0,1}(X)$ into itself. We restrict this endomorphism $K\left(\partial / \partial z_{\alpha}, \partial / \partial \bar{z}_{\beta}\right)$ to $T_{z}^{* 0,1}(X)$ and denote it by $\left.K(\alpha, \bar{\beta})\right)$. By $P_{k}$ we shall denote the group of permutations on $k$-symbols. Let $\rho \in P_{k}$, and $A_{1}, \cdots, A_{k}$ be operators from a suitable space into itself. By $\rho\left(A_{1} \circ \cdots \circ A_{k}\right)$ we shall denote the operator $A_{\rho(1)} \circ \cdots \circ A_{\rho(k)}$. For the sake of simplicity from now on we shall assume that the coordinate functions $z_{1}, \cdots, z_{n}$ are chosen such that $\left(g_{i j}\left(z^{\prime}\right)\right)$ is the identity matrix.

Lemma 5.2. Let $l, m, p, i$ be non-negative integers such that $l+m+p+i$ $=n, \sigma$ be a permutation of $\{1, \cdots, m+p\}, \rho$ be a permutation of $\{1, \cdots, 2 p\}$ and $\tau$ be a permutation of $\{1, \cdots, l+m\}$. Then

$$
\begin{aligned}
& \sum_{1 \leq \alpha_{1}, \ldots, \alpha_{m+p} \leq n} \sum_{q=0}^{n}(-1)^{q} \operatorname{Tr}\left[\left\{\tau \left(\left(D^{q} S\right)^{l} \circ\left(I_{\xi} \otimes D^{q} K\left(\alpha_{1}, \bar{\alpha}_{\sigma(1)}\right)\right) \circ \ldots\right.\right.\right. \\
& \left.\left.\circ\left(I_{\xi} \otimes D^{q} K\left(\alpha_{m}, \bar{\alpha}_{\sigma(m)}\right)\right)\right) \circ \rho\left(V_{\alpha_{m+1}} \circ \nabla_{\bar{\alpha}_{\sigma(m+1)}} \circ \cdots \circ \nabla_{\alpha_{m+p}} \circ \nabla_{\bar{\alpha}_{\sigma}(m+p)}\right)\right\} \\
& \left.\cdot\left(U^{i, q}\left(z^{\prime}, z\right)\right)\right]\left(z^{\prime}, z^{\prime}\right)=\sum_{r=l}^{n} \sum_{\delta \in P_{n-r}} F_{\delta} \sum_{1 \leq \alpha_{1}, \ldots, \alpha_{n-r} \leq n} \\
& \text {. } \sum_{q=0}^{n}(-1)^{q} \operatorname{Tr}\left[\left(D^{q} S\right)^{r} \circ\left(I_{\xi} \otimes D^{q} K\left(\alpha_{1}, \bar{\alpha}_{\dot{\delta}(1)}\right)\right) \circ \cdots\right. \\
& \left.\circ\left(I_{\xi} \otimes D^{q} K\left(\alpha_{n-r}, \bar{\alpha}_{\tilde{\delta}(n-r)}\right)\right)\right]\left(z^{\prime}\right),
\end{aligned}
$$

where $F_{\delta}$ 's are constants depending upon $\delta, l, m, p, i$ and the permutations $\sigma, \rho$.

Proof. We shall prove the lemma by induction on $i$ and $p$. Let $j$ be a nonnegative integer and suppose that the lemma has been proved for $i<j$. We shall prove the lemma for $i=j$. Let $A_{q}$ denote the operator

$$
\begin{array}{cl}
\sum_{1 \leq \alpha_{1}, \ldots, \alpha_{m+p} \leq n} \tau\left(\left(D^{q} S\right)^{l} \circ\left(I_{\xi} \otimes D^{q} K\left(\alpha_{1}, \bar{\alpha}_{\sigma(1)}\right)\right) \circ \ldots \circ\left(I_{\xi} \otimes D^{q} K\left(\alpha_{m}, \bar{\alpha}_{\sigma(m)}\right)\right)\right) & \\
& \circ \rho\left(\nabla_{\alpha_{m+1}} \circ \nabla_{\bar{\alpha}_{\sigma(m+1)}} \circ \ldots \circ \nabla_{\alpha_{m+p}} \circ \nabla_{\bar{\alpha}_{\sigma}(m+p)}\right) .
\end{array}
$$


First suppose that $p=0$. If $j=0$, then the lemma follows from Corollary 2.6 since $U^{0, q}\left(z^{\prime}, z^{\prime}\right)$ is the identity endomorphism. Therefore we can assume that $j>0$.

Applying the operator $A_{q}$ to both sides of (5.1) and then taking the trace we get

$$
\begin{aligned}
j \sum_{q=0}^{n}( & -1)^{q} \operatorname{Tr}\left[A_{q}\left(U^{j, q}\left(z^{\prime}, z\right)\right)\right]\left(z^{\prime}, z^{\prime}\right) \\
= & \sum_{\alpha} \sum_{q=0}^{n}(-1)^{q} \operatorname{Tr}\left[A_{q} \circ \nabla_{\alpha} \circ \nabla_{\bar{\alpha}}\left(U^{j-1, q}\left(z^{\prime}, z\right)\right)\right]\left(z^{\prime}, z^{\prime}\right) \\
& +\sum_{q=0}^{n}(-1)^{q} \operatorname{Tr}\left[A_{q} \circ\left(I_{\xi} \otimes D^{q} \tilde{K}\right)\left(U^{j-1, q}\left(z^{\prime}, z\right)\right)\right]\left(z^{\prime}, z^{\prime}\right) \\
& -\sum_{q=0}^{n}(-1)^{q} \operatorname{Tr}\left[A_{q} \circ D^{q} S\left(U^{j-1, q}\left(z^{\prime}, z\right)\right)\right]\left(z^{\prime}, z^{\prime}\right) .
\end{aligned}
$$

By the induction hypothesis Lemma 5.2 holds for each of the three terms on the right hand side of the above equation. Hence we have (5.2) for $i=j$ and $p=0$.

Now suppose that $p>0$ and the lemma has been proved for smaller values of $p$ (and $i=j$ ). Let $B_{q}, C_{q}$ be respectively the operators

$$
\begin{gathered}
\tau\left(\left(D^{q} S\right)^{l} \circ\left(I_{\xi} \otimes D^{q} K\left(\alpha_{1}, \bar{\alpha}_{\sigma(1)}\right)\right) \circ \cdots \circ\left(I_{\xi} \otimes D^{q} K\left(\alpha_{m}, \bar{\alpha}_{\sigma(m)}\right)\right)\right), \\
\rho\left(\nabla_{\alpha_{m+1}} \circ \nabla_{\bar{\alpha}_{\sigma(m+1)}} \circ \cdots \circ \nabla_{\alpha_{m+p}} \circ \nabla_{\bar{\alpha}_{\sigma}(m+p)}\right),
\end{gathered}
$$

and $X_{1}, \cdots, X_{2 p}$ be vector fields such that

$$
X_{\rho-1(2 k+1)}=\partial / \partial z_{\alpha_{m+k+1}}, 0 \leq k \leq p-1 ; X_{\rho-1(2 k)}=\partial / \partial \bar{z}_{\alpha_{\sigma(m+k)}}, 1 \leq k \leq p .
$$

Then $C_{q}=\nabla_{X_{1}} \circ \cdots \circ V_{X_{2} p}$. As in the proof of Lemma 5.1, we want to apply the operator $A_{q}$ to both sides of (5.1) and then take the trace. We first look at the term $A_{q} \circ \nabla_{r \frac{d}{d r}}\left(U^{j, q}\left(z^{\prime}, z\right)\right)$, and shall apply Lemma 3.3 for the operator $\nabla_{X_{1}} \circ \cdots \circ \nabla_{X_{2}} \circ \nabla_{r \frac{r}{d r}}$. Since the vector field $r \frac{d}{d r}$ is zero at $z^{\prime}$, we have

$$
\begin{aligned}
& {\left[B_{q} \circ \nabla_{r \frac{d}{d r}} \circ \nabla_{X_{1}} \circ \cdots \circ \nabla_{X_{2} p}\left(U^{j, q}\left(z^{\prime}, z\right)\right)\right]\left(z^{\prime}, z^{\prime}\right)=0 \text {, }} \\
& {\left[\sum_{\sigma(2)<\cdots<\sigma(2 p)} B_{q} \circ\left(I_{\xi} \otimes D^{q} K\left(r \frac{d}{d r}, X_{\sigma(1)}\right)\right) \circ \nabla_{X_{\sigma(2)}} \circ \cdots\right.} \\
& \left.\circ \nabla_{X_{\sigma}(2 p)}\left(U^{j, q}\left(z^{\prime}, z\right)\right)\right]\left(z^{\prime}, z^{\prime}\right)=0 \text {. }
\end{aligned}
$$

If $0 \leq r \leq 2 p-1$ and $\sigma$ is a permutation of $\{1, \cdots, 2 p\}$, we obtain, by Cancellation Lemma 5.1, 


$$
\begin{aligned}
\sum_{q=0}^{n}(-1)^{q} \operatorname{Tr}[ & B_{q} \circ\left(\left(\nabla_{X_{\sigma(1)}} \circ \cdots \circ \nabla_{X_{\sigma}(r)} S\left(r \frac{d}{d r}, X_{\sigma(r+1)}\right)\right) \otimes I_{q}\right) \\
& \left.\circ \nabla_{X_{\sigma(r+2)}} \circ \cdots \circ \nabla_{X_{\sigma}(2 p)}\left(U^{j, q}\left(z^{\prime}, z\right)\right)\right]\left(z^{\prime}, z^{\prime}\right)=0,
\end{aligned}
$$

$I_{q}$ denoting the identity endomorphism of $C^{\infty}\left(X, \wedge^{q} T^{* 0,1}(X)\right)$. Also, if $2 \leq r$ $\leq 2 p-1$ and $\sigma$ is a permutation of $\{1, \cdots, 2 p\}$, we have (by Cancellation Lemma 5.1)

$$
\begin{gathered}
\sum_{q=0}^{n}(-1)^{q} \operatorname{Tr}\left[B_{q} \circ D^{q}\left(I_{\xi} \otimes \nabla_{X_{\sigma}(1)} \circ \cdots \circ \nabla_{X_{\sigma}(r)} K\left(r \frac{d}{d r}, X_{\sigma(r+1)}\right)\right)\right. \\
\left.\circ \nabla_{X_{\sigma}(r+2)} \circ \cdots \circ \nabla_{X_{\sigma}(2 p)}\left(U^{j, q}\left(z^{\prime}, z\right)\right)\right]\left(z^{\prime}, z^{\prime}\right)=0 .
\end{gathered}
$$

Let $\left[X_{s}, r \frac{d}{d r}\right]=X_{s}+\tilde{X}_{s}$. Then $\hat{X}_{s}\left(z^{\prime}\right)=0$ and it is an immediate consequence of Lemma 3.3 applied to the vector fields $\bar{X}_{s}, X_{1}, \cdots, X_{s-1}$ and Lemma 5.1 that

$$
\begin{gathered}
\sum_{q=0}^{n}(-1)^{q} \operatorname{Tr}\left[B_{q} \circ \nabla_{X_{1}} \circ \cdots \circ \nabla_{X_{s-1}} \circ \nabla_{\tilde{X}_{s}} \circ \nabla_{X_{s+1}} \circ \ldots\right. \\
\left.\circ \nabla_{X_{2} p}\left(U^{j, q}\left(z^{\prime}, z\right)\right)\right]\left(z^{\prime}, z^{\prime}\right)=0
\end{gathered}
$$

Thus

$$
\begin{aligned}
& \sum_{q=0}^{n}(-1)^{q} \operatorname{Tr}\left[A_{q} \circ \nabla_{r \frac{d}{d r}}\left(U^{j, q}\left(z^{\prime}, z\right)\right)\right]\left(z^{\prime}, z^{\prime}\right) \\
& \quad=2 p \sum_{q=0}^{n}(-1)^{q} \operatorname{Tr}\left[A_{q}\left(U^{j, q}\left(z^{\prime}, z\right)\right)\right]\left(z^{\prime}, z^{\prime}\right)+T
\end{aligned}
$$

where

$$
\begin{aligned}
T=\sum_{\substack{\sigma(1)<\sigma(2) \\
\sigma(3)<\cdots<<(2 p)}} \sum_{q=0}^{n}(-1)^{q} \operatorname{Tr}[ & B_{q} \circ D^{q}\left(I_{\xi} \otimes V_{X_{\sigma(1)}} K\left(r \frac{d}{d r}, X_{\sigma(2)}\right)\right) \\
& \left.\circ V_{X_{\sigma(3)}} \circ \cdots \circ \nabla_{X_{\sigma(2 p)}}\left(U^{j, q}\left(z^{\prime}, z\right)\right)\right]\left(z^{\prime}, z^{\prime}\right) .
\end{aligned}
$$
Since the vector field $r \frac{d}{d r}$ is zero at $z^{\prime}$ for any two vector fields $X$ and $Y$, we
have

$$
\left(\nabla_{X}\left(K\left(r \frac{d}{d r}, Y\right)\right)\right)\left(z^{\prime}\right)=\left(K\left(\left[X, r \frac{d}{d r}\right], Y\right)\right)\left(z^{\prime}\right)=(K(X, Y))\left(z^{\prime}\right) .
$$

Moreover, 


$$
\begin{aligned}
& K\left(\partial / \partial z_{\alpha}, \partial / \partial z_{\beta}\right)=K\left(\partial / \partial \bar{z}_{\alpha}, \partial / \partial \bar{z}_{\beta}\right)=0 \\
& K\left(\partial / \partial z_{\alpha}, \partial / \partial \bar{z}_{\beta}\right)=-K\left(\partial / \partial \bar{z}_{\beta}, \partial / \partial z_{\alpha}\right) .
\end{aligned}
$$

Therefore

$$
\begin{aligned}
T=\sum(-1)^{c(r, s, \rho)} \sum_{q=0}^{n} & (-1)^{q} \operatorname{Tr}\left[B_{q} \circ\left(I_{\xi} \otimes D^{q} K\left(\alpha_{m+r}, \bar{\alpha}_{\sigma(m+s)}\right)\right)\right. \\
& \circ \lambda(r, s, \rho)\left(\nabla_{\alpha_{m+1}} \circ \nabla_{\bar{\alpha}_{\sigma(m+1)}} \circ \cdots \circ \hat{\nabla}_{\bar{\alpha}_{\sigma(m+s)}} \circ \cdots\right. \\
& \left.\left.\circ \hat{\nabla}_{\alpha_{m+r}} \circ \cdots \circ \nabla_{\alpha_{m+p}} \circ \nabla_{\left.\bar{\alpha}_{\sigma(m+p)}\right)}\right)\left(U^{j, q}\left(z^{\prime}, z\right)\right)\right]\left(z^{\prime}, z^{\prime}\right),
\end{aligned}
$$

where $c(r, s, \rho)$ 's are integers depending on $r, s, \rho$, and $\lambda(r, s, \rho)$ 's are permutations depending on $r, s, \rho$. Thus by the induction hypothesis (for $p$ ) $T$ equals the right hand side of (5.2) possibly with different constants $F_{\delta}$ 's depending on $\delta, l, m, p, i, \sigma, \rho$. By Concellation Lemma 5.1, one can easily see that

$$
\begin{gathered}
\sum_{q=0}^{n}(-1)^{q} \operatorname{Tr}\left[A q \circ\left(\Delta_{\bar{z}}\left(r^{2}\right) U^{j, q}\left(z^{\prime}, z\right)\right)\right]\left(z^{\prime}, z^{\prime}\right)=\left(\Delta_{\bar{z}}\left(r^{2}\right)\right)\left(z^{\prime}, z^{\prime}\right) \\
\cdot \sum_{q=0}^{n}(-1)^{q} \operatorname{Tr}\left[A_{q}\left(U^{j, q}\left(z^{\prime}, z\right)\right]\left(z^{\prime}, z^{\prime}\right) .\right.
\end{gathered}
$$

Let

$$
\begin{aligned}
& T_{1}=\sum_{q=0}^{n}(-1)^{q} \operatorname{Tr}\left[A_{q} \circ g^{\beta \alpha} \nabla_{\alpha} \circ \nabla_{\bar{\beta}}\left(U^{j-1, q}\left(z^{\prime}, z\right)\right)\right]\left(z^{\prime}, z^{\prime}\right), \\
& T_{2}=\sum_{q=0}^{n}(-1)^{q} \operatorname{Tr}\left[A_{q} \circ D^{q}\left(I_{\xi} \otimes \tilde{K}\right)\left(U^{j-1, q}\left(z^{\prime}, z\right)\right)\right]\left(z^{\prime}, z^{\prime}\right), \\
& T_{3}=\sum_{q=0}^{n}(-1)^{q} \operatorname{Tr}\left[A_{q} \circ D^{q} S\left(U^{j-1, q}\left(z^{\prime}, z\right)\right)\right]\left(z^{\prime}, z^{\prime}\right) .
\end{aligned}
$$

By Cancellatation Lemma 5.1 we obtain

$$
T_{1}=\sum_{q=0}^{n}(-1)^{q} \operatorname{Tr}\left[A_{q} \circ \nabla_{\alpha} \circ \nabla_{\bar{\alpha}}\left(U^{j-1, q}\left(z^{\prime}, z\right)\right]\left(z^{\prime}, z^{\prime}\right),\right.
$$

and Lemma 3.2 together with Cancellation Lemma 5.1 gives

$$
\begin{aligned}
& T_{2}= \sum_{q=0}^{n}(-1)^{q} \operatorname{Tr}\left[B^{q} \circ D^{q}\left(I_{\xi} \otimes \tilde{K}\right) \circ C_{q}\left(U^{j-1, q}\left(z^{\prime}, z\right)\right)\right]\left(z^{\prime}, z^{\prime}\right) \\
&= \sum_{q=0}^{n}(-1)^{q} \operatorname{Tr}\left[B_{q} \circ D^{q}\left(I_{\xi} \otimes K\left(\partial / \partial z_{\alpha}, \partial / \partial \bar{z}_{\alpha}\right)\right) \circ C_{q}\left(U^{j-1, q}\left(z^{\prime}, z^{\prime}\right)\right)\right]\left(z^{\prime}, z^{\prime}\right), \\
& \text { since } \tilde{K}=K\left(\partial / \partial z_{\alpha}, \partial \bar{z}_{\alpha}\right), \\
& T_{3}=\sum_{q=0}^{n}(-1)^{q} \operatorname{Tr}\left[B_{q} \circ D^{q} S \circ C_{q}\left(U^{j-1, q}\left(z^{\prime}, z\right)\right)\right]\left(z^{\prime}, z^{\prime}\right) .
\end{aligned}
$$


Hence by the induction hypothesis on $i$, each of the terms $T_{1}, T_{2}, T_{3}$ equal to the right hand side of (5.2) possibly with different constants $F_{\delta}$ 's. By applying the operator $A_{q}$ to both sides of equation (5.1) we then have, in view of (5.3), (5.4) and (5.5),

$$
(2 p+j) \sum_{q=0}^{n}(-1)^{q} \operatorname{Tr}\left[A_{q}\left(U^{j, q}\left(z^{\prime}, z\right)\right)\right]\left(z^{\prime}, z^{\prime}\right)+T=T_{1}+T_{2}-T_{3} .
$$

Since we have already proved that each of $T, T_{1}, T_{2}, T_{3}$ equal to the right hand side of (5.2) possibly with different constants $F_{\delta}$ 's and $2 p+j>0$, we obtain (5.2), and the proof of Lemma 5.2 is thus completed.

\section{Proof of Theorem 1.6.}

An immediate consequence of Cancellation Lemma 5.1 is that

$$
\sum_{q=0}^{n}(-1)^{q} \operatorname{Tr} U^{i, q}\left(z^{\prime}, z^{\prime}\right)=0 \quad \text { for } i<n .
$$

Therefore (in view of (4.10)) to complete the proof of Theorem 1.6 and hence that of Theorem 1.1, it is sufficient to prove the following lemma:

Lemma 6.1. The cohomology class $(2 \pi)^{-n}\left(\sum_{q=0}^{n}(-1)^{q} \operatorname{Tr} U^{n, q}\left(z^{\prime}, z^{\prime}\right)\right) e$ equals $[\mathrm{ch}(\xi) \mathscr{T}(X)]_{2 n}$.

We shall prove this lemma in this section. By Lemma 5.2, $\sum_{q=0}^{n}(-1)^{q}$ - Tr $U^{n, q}\left(z^{\prime}, z^{\prime}\right)$ equals the right hand side of (5.2) with constants $F_{\delta}^{\prime}$ 's depending only on the dimension $n$ of the manifold and the permutation $\delta$. Let $\tilde{\varphi}$ and $\varphi$ be the maps as defined in $\S 2$. Then

$$
\begin{aligned}
\left(I_{d} \otimes \varphi\right) S & =\sum S_{b \bar{\alpha}}^{a \bar{\alpha}} g_{\delta \alpha} s_{a} \otimes s_{b} *\left(d z_{\delta} \wedge d \bar{z}_{\beta}\right) \\
& =\sum S_{b \alpha \beta}^{a} s_{a} \otimes s_{b} * \otimes\left(d z_{\alpha} \wedge d \bar{z}_{\beta}\right) \\
\varphi(K(\alpha, \bar{\beta})) & =\sum K_{\bar{\gamma} \alpha \bar{\beta}}^{\bar{\delta}} g_{\varepsilon \delta} d z_{\varepsilon} \wedge d \bar{z}_{\gamma} .
\end{aligned}
$$

Since we have chosen the coordinate functions such that $\left(g_{\alpha \beta}\left(z^{\prime}\right)\right)$ is the identity matrix, at the point $z^{\prime}$ we have

$$
K_{\tilde{\gamma} \alpha \bar{\beta}}^{\bar{\delta}}=K_{\tilde{\gamma} \bar{\beta}}^{\bar{\delta} \alpha}=K_{\bar{\beta} \bar{\delta}}^{\bar{\alpha} \bar{\delta}}=K_{\bar{\beta} \delta \bar{\gamma}}^{\bar{\alpha}},
$$

and therefore

$$
\varphi(K(\alpha, \bar{\beta}))=\sum K_{\bar{\beta} \delta \bar{\gamma}}^{\alpha} d z_{\delta} \wedge d \bar{z}_{\gamma}
$$

Put

$$
S_{b}^{a}=\sum S_{b \alpha \bar{\beta}}^{a} d z_{\alpha} \wedge d \bar{z}_{\beta}, \quad K_{\beta}^{\alpha}=\sum K_{\beta \gamma \bar{\delta}}^{\alpha} d z_{\gamma} \wedge d \bar{z}_{\delta}
$$


Then $\left(S_{b}^{a}\right)$ and $\left(K_{\beta}^{\alpha}\right)$ are local expressions for the curvature forms of hermitian connections in the bundles $\xi$ and $T(X)$ respectively.

By Lemma 5.2 and Corollary 2.6 we get

$$
\begin{aligned}
(2 \pi)^{-n}\left(\sum_{q=0}^{n}(-1)^{q} \operatorname{Tr} U^{n, q}\left(z^{\prime}, z^{\prime}\right)\right) e=\sum_{r=0}^{n} \tilde{\varphi}\left(\sum S_{b \alpha \beta}^{a} s_{a} \otimes s_{b}^{*}\right. \\
\otimes\left(d z_{\alpha} \wedge d \bar{z}_{\beta}\right), \cdots, \sum S_{b \alpha \beta}^{a} s_{a} \otimes s_{b}^{*} \otimes\left(d z_{\alpha} \wedge d \bar{z}_{\beta}\right)(r \text {-times }) \\
\wedge \sum_{\delta \in p_{n-r}} F_{\delta} \bar{K}_{\alpha_{\delta(1)}}^{\alpha_{1}} \wedge \cdots \wedge \bar{K}_{\alpha_{\delta(n-r)} n-r}^{\alpha},
\end{aligned}
$$

where $\bar{K}_{\beta}^{\alpha}$ denotes the conjugate of the form $K_{\beta}^{\alpha}$, and $F_{\delta}$ 's are constants depending on the purmutation $\delta$ and the dimension $n$ of the manifold.

Next we shall express the right hand side of (6.2) in terms of the characteristic classes of $\xi$ and $T(X)$. To this end we start with some preliminaries about characteristic classes, and adopt the definitions and terminology of [2, Chapter XII]. Let $f$ be an invariant homogeneous polynomial function of degree $r$ defined on the Lie algebra $\mathscr{G} l(k, C)$ of $G L(k, C)$. In terms of the canonical basis we can represent an element of the Lie algebra by a matrix $\left(X_{j}^{i}\right)_{1 \leq i, j \leq k}$. Let

$$
f(X)=\sum f_{i_{1} \cdots i_{r}}^{j_{1} \cdots j_{r}} X_{i_{1}}^{j_{1}} \cdots X_{i_{r}}^{j_{r}} .
$$

The constants $f_{i_{1} \cdots i_{r}}^{j_{1} \cdots j_{r}}$ satisfy the condition $f_{i_{(1)} \cdots i_{\sigma(r)}}^{j_{\sigma}(1) \cdots j_{\sigma}(r)}=f_{i_{1} \cdots i_{r}}^{j_{1} \ldots j_{r}}$ for any permutation $\sigma$ of $\{1, \cdots, r\}$. Then the characteristic class $w(f)$ of the vector bundle $\xi$ corresponding to the function $f$ is given by

$$
w(f)=\sum f_{i_{1} \cdots i_{r}}^{j_{1} \cdots j_{r}} S_{i_{1}}^{j_{1}} \wedge \cdots \wedge S_{i_{r}}^{j_{r}} .
$$

Consider the function $f$ defined on the Lie algebra by

$$
f(X)=\operatorname{trace}\left(\exp \left(\frac{i}{2 \pi} X\right)\right)=\operatorname{trace}\left(\sum_{r=0}^{\infty} \frac{i^{r}}{(2 \pi)^{r}} \frac{X^{r}}{r !}\right), \quad X \in \mathscr{G} l(k, C),
$$

and let

$$
f^{r}(X)=\frac{i^{r}}{(2 \pi)^{r} r !} \operatorname{trace} X^{r}
$$

The homogeneous polynomial function $f^{r}$ is invariant by ad $(G L(k, C))$. We shall donote the characteristic class $w\left(f^{r}\right)$ of $\xi$ by $\operatorname{ch}^{r}(\xi)$. By (6.2) we then have

$$
\operatorname{ch}^{r}(\xi)=\frac{i^{r}}{(2 \pi)^{r} r !} \sum_{1 \leq i_{1}<\cdots<i_{r} \leq k} S_{i_{1}}^{i_{2}} \wedge S_{i_{2}}^{i_{3}} \wedge \cdots \wedge S_{i_{r}}^{i_{1}}
$$


The cohomology class $\operatorname{ch}(\xi)=\sum \operatorname{ch}^{r}(\xi)$ is called the Chern character.

\section{Lemma 6.2.}

$$
\begin{gathered}
\tilde{\varphi}\left(\sum_{1} S_{b \alpha \beta}^{a} s_{a} \otimes s_{b}^{*} \otimes\left(d z_{\alpha} \wedge d \bar{z}_{\beta}\right), \cdots,\right. \\
\left.\sum_{r} S_{b \alpha \beta}^{a} s_{a} \otimes s_{b}^{*} \otimes\left(d z_{\alpha} \wedge d \bar{z}_{\beta}\right)\right) \\
=r !(-2 \pi \sqrt{-1})^{r} \operatorname{ch}^{r}(\xi) .
\end{gathered}
$$

Proof. This lemma is an immediate consequence of the definition of $\tilde{\varphi}$ and the definition of Chern character.

Let $f_{0}(X), f_{1}(X), \cdots, f_{n}(X)$ be the polynomial functions defined on the Lie algebra $\mathscr{G} l(n, C)$ of the Lie group $G L(n, C)$ by

$$
\operatorname{det}\left(\lambda I_{n}-\frac{1}{2 \pi \sqrt{-1}} X\right)=\sum_{r=0}^{n} \lambda^{n-r} f_{r}(X)
$$

The polynomial functions $f_{0}, f_{1}, \cdots, f_{n}$ are invariant by ad $(G L(n, C))$. Let $C_{0}, C_{1}, \cdots, C_{n}$ be the characteristic classes $w\left(f_{0}\right), w\left(f_{1}\right), \cdots, w\left(f_{n}\right)$ of the tangent bundle $T(X)$ of $X$ defined by these invariant functions. Then we have

$$
C_{r}(X)=\frac{(-1)^{r}}{(2 \pi \sqrt{-1})^{r} r !} \sum \epsilon_{\sigma} K_{i_{\sigma(1)}}^{i_{1}} \wedge \cdots \wedge K_{i_{\sigma}(r)}^{i_{r}}
$$

where the sum runs over all ordered tuples $\left(i_{1}, \cdots, i_{r}\right)$ and the permutation $\sigma$ of $\{1, \cdots, r\}$, and the symbol $\epsilon_{\sigma}$ denotes the sign of the permutation $\sigma$. $C_{0}(X), C_{1}(X), \cdots, C_{n}(X)$ are the Chern classes of the manifold $X$.

Let $r \leq n$ be a positive integer, and $\delta$ be a permutation of $\{1, \cdots, r\}$. Define a polynomial function $g_{\delta}$ on the Lie algebra $\mathscr{G} l(n, C)$ by

$$
g_{\delta}(X)=\sum_{1 \leq i_{1}<\cdots<i_{r} \leq n} X_{i_{\hat{\delta}(1)}}^{i_{1}} \cdots X_{i_{\delta}(r)}^{i_{r}} .
$$

The polynomial function $g_{\delta}(X)$ is invariant by ad $(G L(n, C))$. By Lemma 2.7 there exists a polynomial $P_{\delta}\left(Y_{1}, \cdots, Y_{r}\right)$ in the variables $Y_{1}, \cdots, Y_{r}$,

$$
P_{\delta}\left(Y_{1}, \cdots, Y_{r}\right)=\sum_{\alpha_{1}+\cdots+r_{r}=r} P_{\delta}^{\alpha} Y_{1}^{\alpha_{1}} \cdots Y_{r}^{\alpha_{r}},
$$

such that

$$
g_{\delta}(X)=P_{\delta}\left(f_{1}(X), \cdots, f_{r}(X)\right), \quad X \in \mathscr{G} l(n, C) .
$$

As an immediate consequence of (6.1), (6.8) and Lemma 6.2 we get

Lemma 6.3. There exist polynomials

$$
P_{r, n}\left(Y_{1}, \cdots, Y_{r}\right)=\sum_{\alpha_{1}+\cdots+r r_{r}=r} P_{r, n}^{\alpha} Y_{1}^{\alpha_{1}} \cdots Y_{r}^{\alpha} r
$$

such that 


$$
(2 \pi)^{-n}\left(\sum_{q=0}^{n}(-1)^{q} \operatorname{Tr} U^{n, q}\left(z^{\prime}, z^{\prime}\right)\right) e=\sum_{r=0}^{n} \operatorname{ch}^{n-r}(\xi) \wedge P_{r, n}\left(C_{1}(X), \cdots, C_{r}(X)\right)
$$

We now proceed to determine the polynomials $P_{r, n}$, and shall prove that $P_{r, n}=P_{r, r}$ and the polynomials $P_{n, n}$ satify the multiplicative property (see $[1, \S 1.1]$ for the definition of multiplicative sequences). We need the following lemmas.

Lemma 6.4. Let $X$ be a complex analytic manifold of dimension $n$, and $z$ a point of $X$. Then there exist a hermitian metric $g$ on $X$ and an open neighbourhood $U$ of $z$ such that the metric $g$ restricted to $U$ is Kaehlerian and such that with respect to the hermitian connection induced by the metric $g,\left(C_{n}(X)\right)(z) \neq 0$ and $\left(C_{i}(X)\right)(z)=0$ for $0<i<n$, the forms $C_{i}(X)$ being defined by (6.6).

Proof. Let $z_{1}, \cdots, z_{n}$ be holomorphic coordinate functions defined in an open neighbourhood $V$ of $z, U$ be an open neighbourhood of $z$ such that the closure of $U$ is contained in $V$, and $\sigma$ be the permutation $\left(\begin{array}{llll}1 & 2 & \cdots & n \\ 2 & 3 & \cdots & 1\end{array}\right)$. There exists a hermitian metric $g$ on $X$ such that restricted to $U, g$ is Kaehlerian and, at the point $z,\left(g_{i j}\right)$ is the identity matrix, $\partial g_{i j} / \partial z_{k}=\partial g_{i j} / \partial \bar{z}_{k}=0$ for all $i, j, k, \partial^{2} g_{i j} / \partial z_{k} \partial \bar{z}_{l}=0$ unless $k=i, l=j$ and the sequence $\{i, j\}$ is a permutation of $\{i, \sigma(i)\}$, and lastly $\partial^{2} g_{i_{\sigma(i)}} / \partial z_{i} \partial \bar{z}_{\sigma(i)}=\partial^{2} g_{\sigma(i) i} / \partial z_{\sigma(i)} \partial \bar{z}_{i}=1$. Then the two-forms $K_{j}^{i}=0$ unless $\{i, j\}$ is a permutation of $\{k, \sigma(k)\}$ for some $k$, and

$$
K_{\sigma(i)}^{i}=-d z_{\sigma(i)} \wedge d \bar{z}_{i}, \quad K_{i}^{\sigma(i)}=-d z_{i} \wedge d \bar{z}_{\sigma(i)}
$$

It can easily be seen that $\left(C_{i}(X)\right)(z)=0$ for $0<i<n$ and $\left(C_{n}(X)\right)(z)$ $=(-2)^{n} \pi^{-n} e(z), e$ being the volume element. This completes the proof of Lemma 6.4.

Similarly, one can prove the following lemma.

Lemma 6.5. Let $X$ be a complex manifold of dimension $n, z$ be an arbitrary point of $X$, and $\xi$ be a holomorphic vector bundle of rank $k, 0<k \leq n$. Then there exist hermitian metrices in $X$ and $\xi$ such that the hermitian metric in $X$ restricted to an open neighbourhood of $z$ is Kaehlerian and such that with respect to the hermitian connections in $T(X)$ and $\xi$ given by these metrices, $\left(C_{i}(X)\right)(z)=0$ for $0<i \leq n$ and $\left(\operatorname{ch}^{k}(\xi)\right)(z) \neq 0$.

Given two multi-indices $\alpha=\left(\alpha_{1}, \cdots, \alpha_{n}\right)$ and $\beta=\left(\beta_{1}, \cdots, \beta_{n}\right)$ we shall say that $\beta<\alpha$ if there exists a positive integer $s$ such that $\beta_{t}=\alpha_{t}$ for $s<t \leq n$ and $\beta_{s}<\alpha_{s}$.

Lemma 6.6. Given a multi-index $\alpha=\left(\alpha_{1}, \cdots, \alpha_{n}\right)$ such that $\sum i \alpha_{i}=n$, there exist a complex analytic manifold $X$, a point $z \in X$ and a hermitian metric $g$ on $X$ such that $g$ is Kaehlerian in an open neighbourhood of $z$ and, at the point $z$, 


$$
C_{1}^{\alpha_{1}}(X) \cdots C_{n}{ }^{\alpha}{ }^{n}(X) \neq 0, \quad C_{1}{ }^{\beta_{1}}(X) \cdots C_{n}{ }^{{ }^{n} n}(X)=0 \text { for } \beta=\left(\beta_{1}, \cdots, \beta_{n}\right)
$$

such that $\sum i \beta_{i}=n$ and $\beta<\alpha$.

Proof. Let the partition $n=1+\cdots+1$ ( $\alpha_{1}$ times $)+2+\cdots+2$ ( $\alpha_{2}$ times $)+\cdots+n+\cdots+n$ ( $\alpha_{n}$ times $)$ be denoted as $n=r_{1}+\cdots+r_{k}$. By Lemma 6.4, there exist a complex manifold $X_{j}$ of dimension $r_{j}$, a point $z_{j}$ of $X_{j}$, and a hermitian metric $g$ on $X_{j}$ such that $g$ is Kaehlerian in a neighbourhood of $z_{j}$ and, at the point $z_{j}$,

$$
C_{i}\left(X_{j}\right)=0 \text { for } 0<i<r_{j}, \quad C_{r_{j}}\left(X_{j}\right) \neq 0 .
$$

Let $X=X_{1} \times \cdots \times X_{k}$ and $z=\left(z_{1}, \cdots, z_{k}\right)$. Then the complex manifold $X$, the point $z$ of $X$, and the hermitian metric on $X$ induced by the hermitian metrices on $X_{j}$ satisfy the requirements of the lemma.

We now introduce some notations. Denote the forms $P_{r, n}\left(C_{1}(X), \ldots, C_{r}(X)\right)$ by $P_{r, n}(X)$, let $Q\left(Y_{1}, \cdots, Y_{m}\right)=\sum_{\alpha_{1}+\cdots+m \alpha_{m}=m} q^{\alpha} Y_{1}^{\alpha_{1}} \cdots Y_{m}^{\alpha_{m}}$ be a polynomial, and make the formal substitution $Y_{i}=\sum_{j+k=i}^{m} Z_{j} X_{k}$. Then there exist unique polynomials $R_{j, k}=\sum_{\substack{\alpha_{1}+\ldots+j \alpha_{j}=j \\ \beta_{1}+\cdots+\beta_{k}=k}} r_{j, k}^{\alpha, \beta} Z_{1}^{\alpha_{1}} \cdots Z_{j}^{\alpha_{j}} X_{1}^{\beta_{1}} \cdots X_{k}^{\beta_{k}}$ such that $Q\left(Y_{1}, \cdots, Y_{m}\right)$ $=\sum_{j+k=m} R_{j, k}\left(Z_{1}, \cdots, Z_{j}, X_{1}, \cdots, X_{k}\right)$. We shall denote the polynomials $R_{j, k}$ by $Q^{j, k}$.

Now we are in a position to prove the following lemma.

Lemma 6.7. Polynomials $P_{n, n}$ satisfy the multiplicative property and $P_{r, n}$ $=P_{r, r}$ for $0 \leq r \leq n$.

Proof. We first observe the following. Let $X$ be a compact complex manifold of dimension $n$, and $g$ a hermitian metric on $X$ such that in a neighbourhood $U$ of a point $z^{\prime}$ of $X, g$ is Kaehlerian. Let $\xi$ be a holomorphic vector bundle over $X$, and $U^{0, q}\left(z^{\prime}, z\right), U^{1, q}\left(z^{\prime}, z\right), \cdots, U^{N, q}\left(z^{\prime}, z\right)$ be $C^{\infty}$-double forms defined in a neighbourhood of the diagonal in $U \times U$ such that $U^{0, q}\left(z^{\prime}, z^{\prime}\right)$ is the identity endomorphism of $\xi_{z^{\prime}} \otimes \wedge^{q} T_{z^{\prime}}^{* 0,1}(X)$ and the forms $U^{i, q}\left(z^{\prime}, z\right)$ constitute the solution of system of equations (4.9). Then Lemmas 5.1, 5.2, 6.2 and 6.3 hold at all points $z^{\prime}$ of $U$.

Let us consider a partition $n=n_{1}+n_{2}, n_{1}$ and $n_{2}$ being positive integers. Let $X_{1}, X_{2}$ be complex manifolds (with hermitian metrices) of dimensions $n_{1}, n_{2}$ respectively. Suppose that there exist points $z_{1} \in X_{1}, z_{2} \in X_{2}$ and open neighbourhoods $U_{1}, U_{2}$ of $z_{1}, z_{2}$ respectively such that the hermitian metrices restricted to $U_{1}, U_{2}$ are Kaehlerian. Let $\xi_{1}$ be a holomorphic vector bundle (with a hermitian metric) on $X_{1}$, and $\xi_{2}$ the trivial line bundle on $X_{2}$. Put $X=X_{1} \times X_{2}$ and $\xi=\xi_{1} \otimes \xi_{2}$, and suppose that $\left\{U_{j}^{j, q}\left\{z_{j}^{\prime}, z_{j}\right)\right\}_{0 \leq i \leq N}(j=1,2)$ constitute the solution of system of equations (4.9) in a neighbourhood of the diagonal in $U_{j} \times U_{j}$ with the initial condition that $U_{j}^{0, q}\left(z_{j}^{\prime}, z_{j}^{\prime}\right)$ be the identity endomorphism. 
Given vector spaces $W, M, M_{1}, M_{2}$ and $N, N_{1}, N_{2}$ such that $M_{1}, M_{2}$ are subspaces of $M$ and $N_{1}, N_{2}$ are subspaces of $N$, there is a natural map from $\left(\left(W \otimes \wedge^{q_{1}} M_{1}\right) \otimes\left(W \otimes \wedge^{q_{1}} N_{1}\right)\right) \times\left(\wedge^{q_{2}} M_{2} \otimes \wedge^{q_{2}} N_{2}\right)$ to $\left(W \otimes \wedge^{q_{1}+q_{2}} M\right) \otimes$ $\left(W \otimes \wedge^{q_{1}+q_{2}} N\right)$ sending $\left(w_{1} \otimes x_{1} \otimes w_{2} \otimes y_{1}, x_{2} \otimes y_{2}\right)$ to $\left(w_{1} \otimes\left(x_{1} \wedge x_{2}\right)\right) \otimes$ $\left(w_{2} \otimes\left(y_{1} \wedge y_{2}\right)\right)$, where $w_{1}, w_{2} \in W, \quad x_{1} \in \wedge^{q_{1}} M_{1}, \quad y_{1} \in \wedge^{q_{1}} N_{1}, \quad x_{2} \in \wedge^{q_{2}} M_{2}$, $y_{2} \in \wedge^{q_{2}} N_{2}$. We shall denote the image of an element $(x, y), x \in\left(W \otimes \wedge^{q_{1}} M_{1}\right)$ $\otimes\left(W \otimes \wedge^{q_{1}} N_{1}\right), y \in \wedge^{q_{2}} M_{2} \otimes \wedge^{q_{2}} N_{2}$ under this map by $x \wedge y$. Then we have

$$
U^{i, q}\left(z^{\prime}, z\right)=\sum_{\substack{j+k=i \\ q_{1}+q_{2}=q}} U_{1}^{j, q_{1}}\left(z_{1}^{\prime}, z_{1}\right) \wedge U_{2}^{k, q_{2}}\left(z_{2}^{\prime}, z_{2}\right),
$$

where $z^{\prime}=\left(z_{1}^{\prime}, z_{2}^{\prime}\right)$ and $z=\left(z_{1}, z_{2}\right)$. Therefore

$$
\begin{aligned}
\sum_{q=0}^{n} & (-1)^{q} \operatorname{Tr} U^{n, q}(z, z) \\
& =\sum_{j+k=n}\left(\sum_{q_{1}=0}^{n_{1}}(-1)^{q_{1}} \operatorname{Tr} U_{1}^{i, q_{1}}\left(z_{1}, z_{1}\right)\right)\left(\sum_{q_{2}=0}^{n_{2}}(-1)^{q_{2}} \operatorname{Tr} U_{2}^{k, q_{2}}\left(z_{2}, z_{2}\right)\right) \\
& =\left(\sum_{q_{1}=0}^{n_{1}}(-1)^{q_{1}} \operatorname{Tr} U_{1}^{n_{1}, q_{1}}\left(z_{1}, z_{1}\right)\right)\left(\sum_{q_{2}=0}^{n_{2}}(-1)^{q_{2}} \operatorname{Tr} U_{2}^{n_{2}, q_{2}}\left(z_{2}, z_{2}\right)\right)
\end{aligned}
$$

by Lemma 5.1 ,

which implies

$$
\begin{aligned}
\left(\sum_{r=0}^{n} \operatorname{ch}^{n-r}(\xi) \wedge P_{r, n}(X)\right)(z)=\left(\prod _ { 1 } ^ { * } \left(\sum_{s=0}^{n_{1}} \operatorname{ch}^{n_{1}-s}\left(\xi_{1}\right)\right.\right. & \left.\left.\wedge P_{s, n_{1}}\left(X_{1}\right)\right)\right)\left(z_{1}\right) \\
& \wedge \prod_{2}^{*}\left(P_{n_{2}, n_{2}}\left(X_{2}\right)\right),
\end{aligned}
$$

where $\prod_{1}, \prod_{2}$ are projections from $X$ onto $X_{1}, X_{2}$. Since

$$
C_{i}(X)=\sum_{j+k=i} \prod_{1}^{*} C_{j}\left(X_{1}\right) \wedge \prod_{2}^{*} C_{k}\left(X_{2}\right) \text { and } \operatorname{ch}^{i}(\xi)=\prod_{1}^{*} \operatorname{ch}^{i}\left(\xi_{1}\right),
$$

we have

$$
\begin{aligned}
& {\left[\sum _ { \substack { r = 0 \\
r _ { 1 } + r _ { 2 } = r } } ^ { n } \prod _ { 1 } ^ { * } ( \operatorname { c h } ^ { n - r } ( \xi _ { 1 } ) ) \wedge P _ { r , n } ^ { r _ { 1 } , r _ { 2 } } \left(\prod_{1}^{*} C_{1}\left(X_{1}\right), \cdots,\right.\right.} \\
& \left.\left.\prod_{1}^{*} C_{r_{1}}\left(X_{1}\right), \prod_{2}^{*} C_{1}\left(X_{2}\right), \cdots, \prod_{2}^{*} C_{r_{2}}\left(X_{2}\right)\right)\right](z) \\
& =\prod_{1}^{*}\left(\sum_{s=0}^{n_{1}} \operatorname{ch}^{n_{1}-s}\left(\xi_{1}\right) \wedge P_{s, n_{1}}\left(X_{1}\right)\right)(z) \wedge \prod_{2}^{*}\left(P_{n_{2}, n_{2}}\left(X_{2}\right)\right)(z) .
\end{aligned}
$$

Let us take $\xi_{1}$ to be the trivial bundle. Then $\operatorname{ch}^{s}\left(\xi_{1}\right)=0$ for $s>0$, and from (6.9) follows

$$
\begin{gathered}
\left(P_{n, n}^{n_{1}, n_{2}}\left(\prod_{1}^{*} C_{1}\left(X_{1}\right), \cdots, \prod_{1}^{*} C_{n_{1}}\left(X_{1}\right), \prod_{2}^{*} C_{1}\left(X_{2}\right), \cdots, \prod_{2}^{*} C_{n_{2}}\left(X_{2}\right)\right)\right)(z) \\
=\prod_{1}^{*}\left(P_{n_{1}, n_{1}}\left(X_{1}\right)\right)(z) \wedge \prod_{2}^{*}\left(P_{n_{2}, n_{2}}\left(X_{2}\right)\right)(z)
\end{gathered}
$$


which and Lemma 6.6 imply

$$
P_{n, n}^{n_{1}, n_{2}}\left(Y_{1}, \cdots, Y_{n_{1}}, Z_{1}, \cdots, Z_{n_{2}}\right)=P_{n_{1}, n_{1}}\left(Y_{1}, \cdots, Y_{n_{1}}\right) \times P_{n_{2}, n_{2}}\left(Z_{1}, \cdots, Z_{n_{2}}\right)
$$

in the polynomial ring $C\left(Y_{1}, \cdots, Y_{n_{1}}, Z_{1}, \cdots, Z_{n_{2}}\right)$. This is precisely what is needed in order that the polynomials $P_{n, n}$ may satisfy the multiplicative property.

Taking $\xi_{1}$ to be a holomorphic vector bundle of rank $n_{1}$, (6.9) together with Lemma 6.5 gives

$$
\left(P_{n_{2}, n}\left(C_{1}\left(X_{2}\right), \cdots, C_{n_{2}}\left(X_{2}\right)\right)\right)\left(z_{2}\right)=\left(P_{n_{2}, n_{2}}\left(C_{1}\left(X_{2}\right), \cdots, C_{n_{2}}\left(X_{2}\right)\right)\right)\left(z_{2}\right),
$$

since $P_{0, n_{1}}=1$. In view of Lemma 6.6 this implies that in the polynomial ring $C\left(Y_{1}, \cdots, Y_{n_{2}}\right)$,

$$
P_{n_{2}, n}\left(Y_{1}, \cdots, Y_{n_{2}}\right)=P_{n_{2}, n_{2}}\left(Y_{1}, \cdots, Y_{n_{2}}\right),
$$

completing the proof of Lemma 6.7.

We now come to the proof of Lemma 6.1.

Proof of Lemma 6.1. In view of Lemmas 6.3 and 6.7 we need only to prove that if $X$ is a complex analytic manifold of dimension $n$, and $r$ is an integer less than or equal to $n$, then $P_{r, r}\left(C_{1}(X), \cdots, C_{r}(X)\right)$ equals the $r$-th component of the Todd class $\mathscr{T}(X)$. In other words, we need only to prove that the polynomials $P_{n, n}$ are Todd polynomials; see [1, $\left.\$ 1.7\right]$ for the definition to Todd polynomials.

By Lemma 6.7, polynomials $P_{n, n}$ enjoy the multiplicative property. Therefore there exists a power series $Q(z)$ which completely determines the polynomials $P_{n, n}$ (see [1, Lemma 1.2.1]), and we need only to prove that $Q(z)$ is the power series $z /\left(1-e^{-z}\right)$ or that the coefficient of $z^{n}$ in $(Q(z))^{n+1}$ is equal to 1 (see [1, Lemma 1.7.1]). For this we consider the complex projective space $P_{n}(C)$. There exists a generator $h_{n} \in H^{2}\left(P_{n}(C), Z\right)$ such that the Chern classes of $P_{n}(C)$ are given by (see [1, Theorem 4.10.2])

$$
C_{i}\left(P_{n}(C)\right)=\left(\begin{array}{c}
n+1 \\
i
\end{array}\right) h_{n}^{i}
$$

The Euler-Poincaré characteristic $\chi(X, \Omega(\xi))$, for $X=P_{n}(C)$ and $\xi$ equal to the trivial bundle over $X$, equals 1 , and $C_{n}\left[P_{n}(C)\right]$, the value of the $n$-th Chern class of $P_{n}(C)$ on the $2 n$-dimensional fundamental cycle of $X$, equals $n+1$. Therefore we have

$$
P_{n, n}\left(\left(\begin{array}{c}
n+1 \\
1
\end{array}\right), \cdots,\left(\begin{array}{c}
n+1 \\
n
\end{array}\right)\right)=1 .
$$

By the factorization

$$
1+\left(\begin{array}{c}
n+1 \\
1
\end{array}\right) z+\cdots+\left(\begin{array}{c}
n+1 \\
n
\end{array}\right) z^{n}+z^{n+1}=(1+z)^{n+1}
$$


and the multiplication property for the polynomials $P_{n, n}$ we thus obtain

$$
P_{n, n}\left(\left(\begin{array}{c}
n+1 \\
1
\end{array}\right), \cdots,\left(\begin{array}{c}
n+1 \\
n
\end{array}\right)\right)=\text { coefficient of } z^{n} \text { in }(Q(z))^{n+1} .
$$

From (6.10) and (6.11) it follows that the coefficient of $z^{n}$ in $(Q(z))^{n+1}$ equals 1. This completes the proof of Lemma 6.1 and hence of Theorem 1.6.

Remark. By an analogous method with essentially different algebraic lemmas from those used in this paper the author has been able to prove the Hirzebruch signature theorem.

\section{References}

[1] F. Hirzebruch, Topological methods in algebraic geometry, Springer, Berlin, 1966.

[2] S. Kobayashi \& K. Nomizu, Foundations of differential geometry, Vol. II, Interscience, New York, 1969.

[ 3 ] V. K. Patodi, Curvature and the eigenforms of the Laplace operator, J. Differential Geometry 5 (1971).

[4] R. T. Seeley, Complex powers of an elliptic operator, Proc. Sympos. Pure Math. Vol. 10, Amer. Math. Soc., 1967, 288-307.

[ 5 ] E. Vesentini, Lectures on Levi convexity of complex manifolds and cohomology vanishing theorems, Tata Institute of Fundamental Research, Bombay, 1967.

Tata Institute Fundamental Research, Bombay 
